

\title{
Detection of the Cosmic Far-Infrared Background in the AKARI Deep Field South
}

\author{
S. Matsuura ${ }^{1}$, M. Shirahata ${ }^{1}$, M. Kawada ${ }^{2}$, T.T. Takeuchi ${ }^{3}$, D. Burgarella ${ }^{4}$, D.L. \\ Clements $^{5}$, W.-S. Jeong ${ }^{6}$, H. Hanami ${ }^{7}$, S.A. Khan ${ }^{8}$, H. Matsuhara ${ }^{1}$, T. Nakagawa ${ }^{1}$, S.

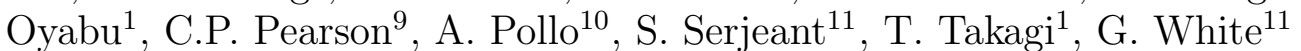 \\ matsuura@ir.isas.jaxa.jp
}

\begin{abstract}
We report the detection and measurement of the absolute brightness and spatial fluctuations of the cosmic infrared background (CIB) with the AKARI satellite. We have carried out observations at 65, 90, 140 and $160 \mu \mathrm{m}$ as a cosmological survey in AKARI Deep Field South (ADF-S), which is one of the lowest cirrus regions with contiguous area on the sky. After removing bright galaxies and subtracting zodiacal and Galactic foregrounds from the measured sky brightness, we have successfully measured the CIB brightness and its fluctuations across a wide range of angular scales from arcminutes to degrees. The measured CIB brightness is consistent with previous results reported from $\mathrm{COBE}$ data but significantly higher than the lower limits at 70 and 160 $\mu \mathrm{m}$ obtained with the Spitzer satellite from the stacking analysis of $24-\mu \mathrm{m}$ selected sources. The discrepancy with the Spitzer result is possibly due to a new galaxy population at high redshift obscured by hot dust. From power spectrum analysis at $90 \mu \mathrm{m}$, three components are identified: shot noise due to individual galaxies; Galactic cirrus emission dominating at the largest angular scales of a few degrees; and an additional component at an intermediate angular scale of $10-30$ arcminutes, possibly due to galaxy clustering. The spectral shape of the clustering component at $90 \mu \mathrm{m}$ is very similar to that at longer wavelengths as observed by Spitzer and BLAST. Moreover, the color of the fluctuations indicates that the clustering component is as red as Ultra-luminous infrared galaxies (ULIRGs) at high redshift, These galaxies are not likely to be the majority of the CIB emission at $90 \mu \mathrm{m}$, but responsible for the clustering component. Our results provide new constraints on the evolution and clustering properties of distant infrared galaxies.
\end{abstract}

Subject headings: cosmology: general — observation: infrared, background radiation — satellite: AKARI - galaxy: formation, clustering

\footnotetext{
${ }^{1}$ Institute of Space and Astronautical Science, JAXA, 3-1-1 Yoshinodai, Sagamihara, Kanagawa 229-8510, Japan

${ }^{2}$ Graduate School of Science, Nagoya University, Furocho, Chikusa-ku, Nagoya 464-0021, Japan

${ }^{3}$ Institute for Advanced Research, Nagoya University, Furo-cho, Chikusa-ku, Nagoya 464-8601, Japan

${ }^{4}$ Laboratoire d'Astrophysique de Marseille, Observatoire Astronomique Marseille Provence, Universite d'AixMarseille, CNRS

${ }^{5}$ Physics Department, Imperial College London, Prince Consort Road, London SW7 2AZ, UK

${ }^{6}$ Space Science Division, Korea Astronomy and Space Science Institute, 61-1, Whaam-dong, Yuseong-gu, Deajeon, 305-348, South Korea

${ }^{7}$ Physics section, Faculty of Humanities and Social Sci-
}

\footnotetext{
ences, Iwate University, Japan

${ }^{8}$ Shanghai Key Lab for Astrophysics, Shanghai Normal University, Shanghai 200234, China

${ }^{9}$ Space Science and Technology Department, Rutherford Appleton Laboratory, Chilton, Didcot, Oxfordshire OX11 OQX, UK

${ }^{10}$ The Andrzej Soltan Institute for Nuclear Studies, ul. Hoza 69, 00-681 Warszawa, Poland

${ }^{11}$ Department of Physics and Astronomy, Open University, Walton Hall, Milton Keynes MK7 6AA, UK
} 


\section{Introduction}

Since the Cosmic Infrared Background (CIB) was observed by the COBE satellite, it has been known that a large fraction of radiation energy in the Universe is released in the infrared (Puget et al. 1996; Hauser et al. 1998). A compelling explanation for the CIB in the far-infrared is the thermal emission of interstellar dust associated with high-redshift galaxies, heated by the internal UV and optical radiation from young stars and active galactic nuclei (AGN), while the nearinfrared background is dominated by starlight from extragalactic sources (Hauser \& Dwek 2001; Kashlinsky 2005; Lagache et al. 2005). Thus, the far-infrared CIB is a powerful probe of dust enshrouded star-formation and AGN activity. Measuring the CIB in the far-infrared may constrain dust emission powered by pre-galactic objects at very high redshift (Coorav \& Yoshida 2004), such as the first generation of stars (Bond et al. 1986; Santos et al. 2002), which are expected to contribute to the near-infrared excess of CIB (Matsumoto et al. 2005).

In Figure 1 we summarize the results of the CIB measurements to date over the entire infrared and submillimeter wavelength range from previous space missions; COBE, IRTS, ISO (Hauser et al. 1998; Fixsen et al. 1998; Finkbeiner et al. 2000; Matsumoto et al. 2005; Juvela et al. 2009). For comparison, we also show various foreground emission components; zodiacal light (sunlight scattered by interplanetary dust), zodiacal emission (thermal emission from interplanetary dust), Galactic light (unresolved stars), diffuse Galactic light (starlight scattered by interstellar dust) and Galactic cirrus emission (thermal emission from interstellar dust), and the CMB. Note at midinfrared wavelengths it is currently impossible to detect the CIB because the zodiacal emission is too bright. In the near-infrared and far-infrared, the foreground emission is relatively weak, and careful modeling and subtraction of the foreground enables one to extract the CIB from the measured sky brightness.

As seen in Figure1, the CIB spectrum at wavelengths longer than $200 \mu \mathrm{m}$ has been well constrained with the FIRAS instrument on COBE (Puget et al. 1996; Fixsen et al. 1998). However, results of the photometric measurements at wave- lengths shorter than $200 \mu \mathrm{m}$ with the DIRBE instrument on COBE are divergengent in the mean levels of the CIB brightness, mainly due to the strong and uncertain foreground contamination of zodiacal emission, which dominates the sky brightness over all of the sky even though the Galactic foreground may be sufficiently weak in low cirrus regions (Hauser et al. 1998; Lagache et al. 2000a; Finkbeiner et al. 2000). Although the CIB brightness was recently estimated using the ISOPHOT data, independently from the COBE data, the 90 $\mu \mathrm{m}$ data gave only an upper limit, and the measurement accuracy of the CIB brightness in the $150-180 \mu \mathrm{m}$ range was in fact worse than that of COBE (Juvela et al. 2009). Figure 1 clearly shows a wavelength desert of the CIB measurement at shorter far-infrared wavelengths, i.e., $<200 \mu \mathrm{m}$. Hence, new measurements of the mean level of CIB are demanded in this region.

In the last decade, many observational efforts have been made to resolve the CIB into individual galaxies by far-infrared deep surveys with infrared space telescopes such as ISO, Spitzer and AKARI (Kawara et al. 1998; Puget et al. 1999; Matsuhara et al. 2000; Kawara et al. 2004; Dole et al. 2004; Fraver et al. 2006a; Matsuura et al. 2007; Shirahata et al. 2008), and consequently the origin of CIB has become clear. As shown in Figure 1. however, the detected galaxies account for only a small fraction $(\sim 10 \%)$ of the measured CIB brightness in the far-infrared. Frayer et al. (2006b) claimed that they resolved more than half of the model CIB at $70 \mu \mathrm{m}$ into point sources in a single deep survey towards the GOODS-N field. In the mid-infrared, a lower limit of the CIB at $24 \mu \mathrm{m}$ is derived from the integrated number counts, and this is thought to account for $~ 70 \%$ of the model CIB at $24 \mu \mathrm{m}$ (Papovich et al. 2004). Dole et al. (2006) obtained lower limits for the CIB at 70 and $160 \mu \mathrm{m}$ by stacking analysis of 24$\mu \mathrm{m}$ detected sources, finding that the mid-infrared sources contribute $\sim 80 \%$ of the CIB in the farinfrared, shown in Figure 1 by the dotted line. In the submillimeter range, similar stacking analysis of $24-\mu \mathrm{m}$ galaxies against the deep surveys at 250, 350 and $500 \mu \mathrm{m}$ by the Balloon-borne LargeAperture Submillimeter Telescope (BLAST) experiment resulted in the conclusion that the $24-\mu \mathrm{m}$ sources produce almost all the CIB in the submillimeter measured with FIRAS (Devlin et al. 2009; 
Marsden et al. 2009). Although these studies using the Spitzer $24-\mu \mathrm{m}$ surveys provided strong constraints on the mean CIB level, the current limit of direct measurement of CIB as diffuse emission in the far-infrared range is still high enough to allow the existence of new population.

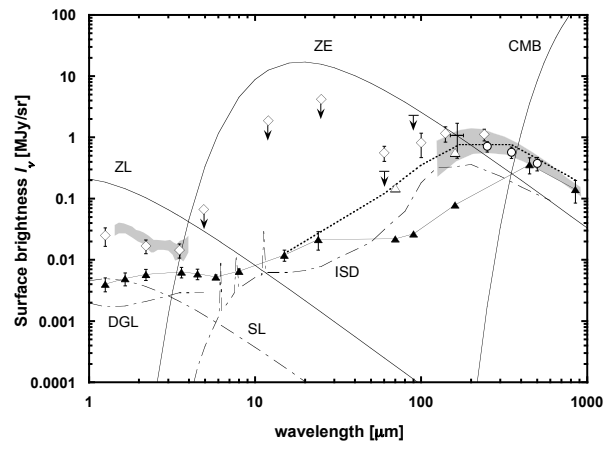

Fig. 1.- Summary of the results of previous CIB measurements in the infrared and submillimeter wavelength range with space missions; COBE/DIRBE (diamonds) (Hauser et al. 1998; Finkbeiner et al. 2000), COBE/FIRAS (shaded region in the submillimeter) (Fixsen et al. 1998), IRAS and ISO (thin line with downward arrows at 60 and $90 \mu \mathrm{m}$ and cross in 150-180 $\mu \mathrm{m}$ ) (Juvela et al. 2009), and IRTS (shaded region in the near-infrared) (Matsumoto et al. 2005). For comparison, we also show various foreground emission components at dark sky; zodiacal light (ZL), zodiacal emission (ZE), Starlight (SL, $K>9$ mag), Diffuse Galactic light (DGL) and Galactic cirrus (ISD), and CMB. The integrated flux from the galaxy counts by deep surveys from the ground in the near-infrared and submillimeter and with the space telescopes in the mid- and farinfrared; ISO, Spitzer and AKARI (Kawara et al. 1998; Puget et al. 1999; Matsuhara et al. 2000; Kawara et al.|2004; Dole et al. 2004; Fraver et al. 2006a; Matsuura et al. 2007; Shirahata et al. 2008), are indicated by the filled triangles connected with thin lines. Stacking of the $24 \mu \mathrm{m}$ galaxies for the Spitzer/MIPS map (open triangles) and the BLAST map (open circles) results in good agreement with the predicted CIB level from a galaxy evolution model by Lagache et al. (2004) (dotted line).
Measuring the spatial fluctuations (anisotropy) of the CIB is a powerful method to investigate the unresolved galaxy population below the detection limit with little contamination from the foreground. The angular power spectrum of the CIB fluctuations is an important observable to trace the distribution of star-forming galaxies with respect to the clustering bias in dark matter halos. The fluctuation measurement is especially effective at longer wavelengths, where direct measurement of the clustering of resolved galaxies is hampered by galaxy confusion due to the diffractionlimited resolution of current telescopes. In fact, power spectrum analysis of the CIB fluctuations has already been done for large area surveys in low cirrus regions; the FIRBACK survey at $170 \mu \mathrm{m}$ by ISOPHOT (Puget et al. 1999; Lagache et al. $2000 \mathrm{~b})$, the MIPS/Spitzer data at $160 \mu \mathrm{m}$ in the SWIRE/GTO field (Lagache et al.|2007), and the BLAST experiment (Viero et al. 2009). These results provided us with useful constraints on galaxy evolution and clustering. Information on the spectral energy distribution (SED) of the CIB fluctuations would be a further important clue in the investigation of the CIB properties. However, the quality of previous CIB fluctuation measurements at shorter far-infrared wavelengths, such as the ELAIS/Lockman survey at $90 \mu \mathrm{m}$ by ISOPHOT (Matsuhara et al. 2000) and new analysis of the IRAS map at $60 \mu \mathrm{m}$ (Miville-Deschênes et al. 2007), is not sufficient to add new information over the MIPS and ISOPHOT results at 160 and $170 \mu \mathrm{m}$.

In this work we aim to make the best measurements of the absolute brightness and fluctuations of the CIB since COBE, especially in the shorter far-infrared wavelength range which is as yet not extensively explored. This measurement is achieved using the high sensitivity of AKARI for diffuse far-infrared emission, high angular resolution, and intensive selection of the survey field that provides the minimum foreground contamination. In the next two sections we describe the observational method and the data processing to produce the final calibrated image of the diffuse emission. In Section 4, we describe how the foreground subtraction so that the absolute brightness of CIB remains as an isotropic residual. In Section 5 , we describe the power spectrum analysis of the final image used to decompose the CIB fluctua- 
tions into galactic and extragalactic components. In Section 6, we show the results of the data analysis compared with previous studies of the CIB with scientific discussion.

\section{Observations}

\subsection{Field selection}

The AKARI observations of the CIB were carried out for a far-infrared cosmological survey program (PI: S. Matsuura) as one of core scientific programs of the AKARI project, known as Mission Programs (MP) (Matsuhara et al. 2006). The survey field named the AKARI Deep Field South (ADF-S) is one of the lowest cirrus regions with contiguous area on the sky (Schlegel et al. 1998) with an area of 12 square degrees near the South Ecliptic Pole; the field center is located at $\mathrm{RA}=4^{h} 44^{m} 00^{s}, \mathrm{DEC}=-53^{\circ} 20^{\prime} 00^{\prime \prime}$ (J2000). The survey field includes a nearby cluster of galaxies, DC 0428-53/Abell S0463 (Dressler 1980). The observation log of this survey is summarized in Table 1 .

The ADF-S field is one of the the best cosmological windows because of its low foreground emission; not only a minimum of galactic foreground, but also low zodiacal background. High visibility of this field for a polar-orbiting satellite such as AKARI is also important to achieve high sensitivity from long exposures. By comparison, the Lockman hole, the most popular low cirrus region as a cosmological window, has a relatively high zodiacal brightness and low visibility due to its low ecliptic latitude.

\subsection{AOT and parameters}

In order to make a mosaic map of the entire field, a total of 200 slow-scan observations were obtained by combination of the Astronomical Observation Templates (AOTs), FIS-01 and FIS02 (Kawada et al. 2007; Matsuura et al. 2007). These AOTs provide for multiple pointings as footprints of the field-of-view (FOV) along a scan path. The area covered by single observation of FIS-01 and FIS-02 is $12 \operatorname{arcmin} \times 0.6 \mathrm{deg}$ and 8 arcmin $\times 1.25 \mathrm{deg}$, respectively, for an exposure time of 10 minutes. Data in four photometric bands, centered at 65, 90, 140 and $160 \mu \mathrm{m}$, were simultaneously taken by a single observation, and the same sky is observed in all the bands except for a small difference in the FOV.

The AOTs include the calibration sequence, i.e. dark current measurement by closing the cold shutter and responsivity check with an internal calibrator, during maneuvers before and after the observation and at the turning points of the roundtrip scans. Such a highly redundant calibration sequence enabled us to correct any responsivity change, referring to astronomical calibration data taken by the same AOT. After the first maneuver to the target position, the cold shutter was opened, the sky signals are monitored during a settling time for the attitude control while the telescope's line of sight is oscillating around the target position, and then the slow-scan observation starts. The signal monitoring before the observation is useful to check for any signal drift including time variation of any stray light (which is described in the next section).

In order to produce a mosaiced image of the entire field with long-strip image segments, a total of 200 slow-scan observations with field overlaps of half a FOV in the cross-scan direction were carried out. The survey was completed in one and a half years over three observational seasons as summarized in Table 1]. Mosaicking of the data taken in different seasons is not straightfoward, and required careful treatment for the time variation of the zodiacal brightness.

\subsection{Supplemental observations}

In addition to the ADF-S survey, supplementary observations using AOT FIS-01 were also made over small areas towards the Lockman Hole (LH) and the North Ecliptic Pole (NEP), as shown in Table 1. One of the LH observations was a mini survey to map over $\sim 1$ sq.deg for the performance verification (PV) soon after the launch of AKARI (Matsuura et al. 2007), and another was included in the same MP program as the ADF-S to measure the galaxy confusion and to check the reproducibility of the measurement a year later. The NEP observation was a Director's Time program to evaluate the confusion noise in a relatively higher cirrus region compared to the ADF-S, the lowest cirrus region. As described in the latter sections, these observations are useful to check the validity of the foreground estimate and to evaluate the cirrus contribution to the sky brightness. 


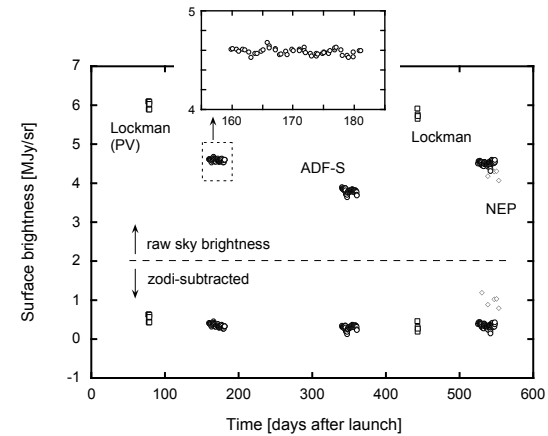

Fig. 2.- Sky brightness at $65 \mu \mathrm{m}$ in ADF-S, Lockman hole and NEP fields, measured in various seasons (upper data points) and the residual brightness after subtraction of the zodiacal foreground (lower data points). The sky brightness values are only for those dates when the fields were observed, rather than the whole length of the mission. The inset shows a magnified view of the data boxed with the dotted line.

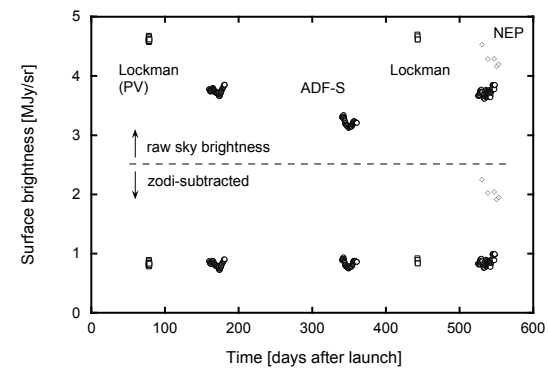

Fig. 3.- Sky brightness at $90 \mu \mathrm{m}$ measured in various fields same as Figure 2

\section{Data processing and calibration}

In this section, we describe the methods of data reduction and analysis of the ADF-S data. The time series data are processed into a co-added image, using the FIS slow-scan analysis tool (SSTool, Ver.20070914) (AKARI FIS Data Users Manual 2007). The SS-Tool includes dark current subtraction, absolute calibration, flat fielding, stray light removal, and co-addition of multiple scans. The SS-Tool is described in detail in Matsuura et al. (2007), therefore we describe them only briefly in the following sections.

\subsection{Time domain analysis}

The raw output of the FIS instrument is time series data, consisting of integration ramps for each detector pixel. Using the SS-Tool, we corrected the raw ramps for non-linearity and converted them to the average current values after removing spikes and glitches caused by cosmic-ray events and any other discontinuities. The SS-Tool process makes a dark current subtraction using the shutter-closed data before and after each observation.

The detector output changes with time depending only on the sky brightness during the scan observation. However, the output changes even when the telescope is pointed towards almost the same sky. This time varying component was identified as being from stray light caused by the Earth's limb emission illuminating the telescope baffle. The stray light appears in the case when the Earth avoidance angle of the telescope is smaller than 80 degrees. The Earth avoidance angle changes with time depending on the orbital phase of the satellite and also with terrestrial season according to the inclination of the orbit even when the same field is being observed. The stray light appears typically for $\sim 150 \mathrm{~s}$ at the begin and end of the observation time. At larger Earth avoidance angles stray light appears for $\sim 200 \mathrm{~s}$ in the mid of the observation time.

Stray light is removed in time domain by highpass filtering the data with a window size of 90 $\mathrm{s}$, which was determined so to remove the slowest signal from the stray light but not to lose faster signals caused by astronomical emission. For the slow-scan observation, the time domain filter is equivalent to a spatial filter in the scan direction. We determined the window size of the filter in the scan direction as to be equivalent to the FOV size in the cross-scan direction, to ensure isotropy.

\section{2. $\quad$ Flat fielding}

Flat-fielding, i.e., correction of responsivity inhomogeneity in detector array, was carried out by measuring the mean brightness of the scanned 
area for each observation. This method is based on the assumption that the sky brightness averaged over the observed area is the same for all array pixels. Accordingly, this method is effective especially for dark sky dominated by zodiacal emission, whose spatial fluctuation is known to be very small from mid-infrared observations; their smoothness in arcmin scales being better than 1\% (Abraham et al. 1998).

According to the results of previous far-infrared missions, e.g., IRAS and COBE, the sky brightness towards high galactic latitudes in the shorter AKARI bands at 65 and $90 \mu \mathrm{m}$ should be dominated by zodiacal emission even at high ecliptic latitude; the expected fractions are $90 \%$ at $65 \mu \mathrm{m}$ and $70 \%$ at $90 \mu \mathrm{m}$, while at 140 and $160 \mu \mathrm{m}$, Galactic cirrus emission is more likely the major $(>50 \%)$ component in many cases. Since the smoothness of Galactic cirrus depends on the angular scale and mean brightness, the flat fielding in cirrus regions is not so excellent as the method using the zodiacal emission. If we choose a sky with a brightness of about $10 \mathrm{MJy} \mathrm{sr}^{-1}$, the smoothness of the cirrus can be better than $10 \%$.

In order to construct a flat-field template for the short wavelength bands, we have carried out observations of zodiacal emission at ecliptic latitudes lower than $20 \mathrm{deg}$ in the performance verification $(\mathrm{PV})$ phase. For the long wavelength bands, we have also taken cirrus observations at intermediate galactic latitudes of about $20 \mathrm{deg}$. However, the use of such a pre-measured flat has suffered from the responsivity change induced by radiation effects and the degradation of detector properties, which may not be perfectly corrected.

For the ADF-S survey, in fact, flat fielding with the observed sky itself has yielded much better results than the pre-measured flat, because the responsivity change could be effectively corrected by the calibration sequence made during each observation. Thus, we constructed such "self-flat" data by averaging the time series data for each pixel after a 3-sigma rejection in time during the settling time for attitude control and the initial scan observation. The flat-field accuracy with this method was generally better than $5 \%$, and the actual effect of the flat field error for each observation was taken into account in the noise map derived from the signal variance for multiple scans over the same sky.

\subsection{Absolute calibration}

\subsubsection{Pre-flight measurement in laboratory}

Pre-flight measurements of the absolute gain including the detector responsivity were carried out in all the wave bands of AKARI in the laboratory, using an external blackbody source at various temperatures ranging from $17 \mathrm{~K}$ to $30 \mathrm{~K}$ with attenuators of $20-30 \mathrm{~dB}$. The measured gain factors show good agreement with each other to within $\pm 5 \%$, and we adopted a mean value as the final gain factor.

In order to compare any measurements done at different times and different conditions either in orbit or in the laboratory, we have corrected the absolute gain value by referring to internal calibrator signals. The reproducibility of the calibrator signals was approximately $\pm 5 \%$, and this was also the limiting factor of the calibration accuracy. In the short wavelength bands at 60 and $90 \mu \mathrm{m}$, we estimated the accuracy of the pre-flight calibration to better than $\pm 7 \%$, by combining the above uncertainties quadratically.

While the monolithic Ge:Ga detectors for the short wavelength bands had fairly good uniformity of the responsivity over the array and showed a high stability in orbit, the stressed Ge:Ga detector arrays for the long wavelength bands showed considerably larger responsivity differences among pixels and strong radiation effects in orbit. These properties made it difficult to correct for the responsivity in the long wavelength bands even with the internal calibrator (c.f. flat field error) and caused additional calibration errors of $\pm 6 \%$ and $\pm 9 \%$ at 140 and $160 \mu \mathrm{m}$, respectively.

\subsubsection{In-orbit measurement}

In order to measure the absolute gain of the detectors in orbit, we carried out observations of diffuse emission whose brightness had been previously accurately measured with $\mathrm{COBE}$, as described in the last section.

For the short wavelength bands, we observed the zodiacal emission at low ecliptic latitudes. To derive the gain factor, we compared our observed data with the COBE/DIRBE model of zodiacal emission, which is reliable at low ecliptic latitude to an accuracy better than 2\% Kelsall et al. 1998). In order to use this method avoiding 
the contamination from Galactic and extragalactic emission, we differentiated the sky brightness measured at different ecliptic latitudes in low cirrus regions with similar HI column densities $\left(\mathrm{N}_{H I} \sim 2 \times 10^{19} \mathrm{~cm}^{-2}\right)$ and compared the differential data with the zodiacal light model. The accuracy of this method is consequently limited by the calibration uncertainty of DIRBE $\sim 10 \%$ (Hauser et al. 1998).

For the long wavelength bands, we observed Galactic cirrus and compared the measured brightness with the COBE/DIRBE annual average map (AAM). The sky brightness at low galactic latitudes in this wavelength range is dominated by Galactic cirrus, seasonal variation of zodiacal emission is smaller than $3 \%$, and the AAM map is thus valid for this calibration. In addition to the calibration uncertainty of DIRBE, the low-frequency noise and instability of the detector during the observations increased the uncertainties to $\pm 15 \%$ and $\pm 19 \%$ at 140 and 160 $\mu \mathrm{m}$, respectively.

\subsubsection{Final gain factor and uncertainty}

For the short wavelength bands, the absolute gain factor of AKARI from pre-flight measurements showed excellent agreement with that from the in-orbit calibration. Their difference was only $5 \%$ at both 65 and $90 \mu \mathrm{m}$. It is noteworthy that this difference is smaller than either of the uncertainties of the pre-flight calibration and the inorbit calibration. For the long wavelength bands, the pre-flight measurement gave a slightly different gain from that of the in-orbit calibration; the difference was $13 \%$ and $25 \%$ at 140 and $160 \mu \mathrm{m}$, respectively, but they agreed with each other within the uncertainties.

In this work, we adopted our own gain factor from the pre-flight measurement to indicate the final result of the CIB brightness. For the foreground subtraction, however, we adopted the inorbit calibration factor to compare our data directly with the DIRBE result. In summary, the absolute calibration uncertainties of our CIB measurements taking all systematics into account are $\pm 7 \%$ at both 65 and $90 \mu \mathrm{m}, \pm 9 \%$ at $140 \mu \mathrm{m}$, and $\pm 13 \%$ at $160 \mu \mathrm{m}$. These numbers are sufficiently small enough for any significant detection of the CIB. More details about the absolute calibration are described in a separate forthcoming paper (S.
Matsuura et al. 2010, in preparation).

\subsection{Co-added image}

Each slow-scan observation covers only a small patch of the sky corresponding to the scanned area. To produce the final mosaic image of the entire ADF-S field from the multiple scans, we coadded the time-series data in grid cells of the sky coordinates, according to the sky position of each detector pixel at each time of the data sampling. The co-addition process was done after subtracting the zodiacal foreground, which showed a seasonal variation as described in the next section.

Even after subtraction of the zodiacal foreground, the mosaic image shows stripes as a scanning effect due to imperfect correction of the detector responsivity. In order to de-stripe the image, we determined the baseline levels of the zodisubtracted images taken in the same season by a smooth filtering in the ecliptic longitude (crossscan) direction with a filter width of 30 arcmin, which corresponds to three times the scan width. This filter works as a notch filter to reduce the noise only at the angular scale of the scan width preserving the fluctuation power at other angular scales. The baseline levels are then subtracted from the zodi-subtracted time-series data.

Finally, we produced the co-added image by calculating the weighted mean after a $3 \sigma$ rejection in each grid cell of the image. We estimated the standard error of the mean value from the standard deviation and number of data samples. The size of the grid cell is determined so as to contain sufficient number of data samples, according to the data interval, the scan speed, number of the scans, and number of the array pixels seeing the same sky. For the ADF-S survey, the grid cell size was set to be $20 \operatorname{arcsec} \times 20$ arcsec each containing more than 8 data samples on average.

\section{Foreground subtraction}

In order to produce the final mosaic image of the entire field by the co-addition of the time series data, we have adjusted the mean sky levels of multiple observations by subtracting the zodiacal foreground with its seasonal variation. For accurate measurements of the CIB, estimates of the contribution from Galactic cirrus and external galaxies are also essential. In this section, we 
describe our methods of the foreground subtraction and show the final mosaic image of the entire ADF-S field.

\subsection{Zodiacal emission}

\subsubsection{Seasonal variation of zodiacal brightness}

Although the seasonal variation of the zodiacal brightness is sometimes an obstacle, it is rather useful to know the contribution of dust near the Earth to the zodiacal brightness. In fact, conventional models of the zodiacal cloud on the basis of the IRAS and COBE observations have been constructed relying on the local dust density and temperature derived from the seasonal variation (Reach 1988; Kelsall et al. 1998). The amplitude of the seasonal variation is not affected by any uncertainty from the isotropic component of the zodiacal emission.

In Figure 2, the measured sky brightness at 65 $\mu \mathrm{m}$ at various locations in the ADF-S are plotted as a function of days after launch. Figure 3 is the same plot but at $90 \mu \mathrm{m}$. The sky brightness values are only for those dates when the field were observed, rather than the whole length of the mission. Statistical errors of the sky brightness from the RMS fluctuation of the image are of the order of a few $\mathrm{kJy} \mathrm{sr}^{-1}$ (smaller than the size of the plot symbols). The sky brightness in the second season in Winter, about a year after launch, is significantly lower than that in the first and third seasons in Summer, as expected from a one-year period of the seasonal variation of zodiacal brightness.

For comparison, the sky brightness towards the LH and NEP, which have been measured as supplemental observations in previously well explored fields, are also shown in Figure 2 and Figure 3 The LH for both PV and MP are brighter than the ADF-S because of the fields higher zodiacal brightness in spite of the similar density of Galactic cirrus. The NEP is brighter than the ADF-S due to the larger contribution from Galactic cirrus even though it has the lowest zodiacal brightness.

\subsubsection{Residual brightness after zodi-subtraction}

To measure the absolute brightness of the CIB, we subtracted the zodiacal foreground for each observation by using the same COBE/DIRBE model as we used for the in-orbit calibration. The zodi-

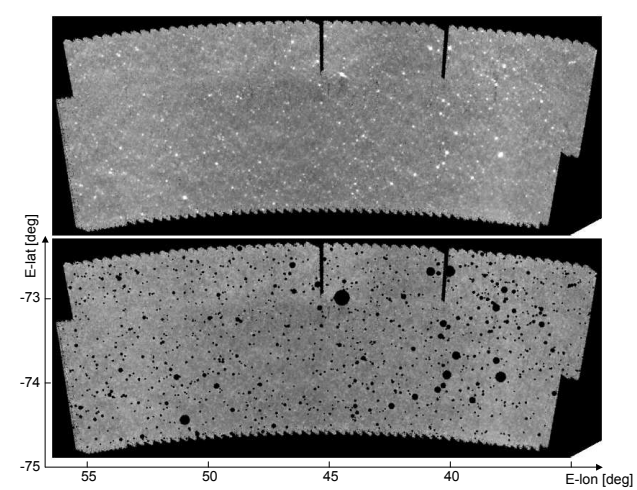

Fig. 4.- Upper: Diffuse map of ADF-S at 90 $\mu \mathrm{m}$ after subtraction of the zodiacal foreground, drawn as linear contours. Brighter the contour, higher the surface brightness. The map size is approximately $6 \mathrm{deg} \times 2 \mathrm{deg}$. Lower: The source removed map after masking out the sources down to $\sim 20 \mathrm{mJy}$. The masked area is filled with zeros to show the source locations clearly.

acal brightness in the AKARI bands were calculated by interpolation from the DIRBE bands with the color correction assuming a blackbody spectrum. The zodi-subtracted data are composed of just the Galactic cirrus and CIB, as long as the zodi-model is correct.

In Figure 2 and Figure 3, residuals after subtraction of the zodiacal foreground are compared with the raw data. The zodi-subtracted data for the ADF-S taken in different seasons show good agreement with each other, not only in the the mean levels but also in the detailed structure mainly due to Galactic cirrus. In addition, the residuals for the ADF-S and LH agree with each other except for the detailed structure, while the NEP is much brighter than the others, especially at $90 \mu \mathrm{m}$ due to the larger contribution from the Galactic cirrus, as described later. At $65 \mu \mathrm{m}$ the difference of the NEP brightness to the other fields is not so clear since the sky brightness is dominated by the zodiacal emission. The results indicate that the zodi-model is correct to an accuracy better than $5 \%$, which is a typical value of the model uncertainty (Kelsall et al. 1998). 


\subsubsection{Zodi-subtracted diffuse map}

The upper image of Figure 4 shows the final diffuse map at $90 \mu \mathrm{m}$ produced by mosaicing the zodi-subtracted data. To obtain this mosaic image, destriping was made by first calculating the mean sky levels of the image strips taken by multiple scans after the removal of bright sources by a 3sigma rejection method, followed by time-domain smooth filtering to the mean sky levels for the multiple scans to correct any slight responsivity difference that may not have been perfectly corrected by the calibration sequence. Finally all images are coadded. The window size used for the time-domain filter corresponds to three scan stripes in the space domain. A large contiguous area was successfully mapped, except for small lost data areas such as the inlets in the upper part of the map caused by problems with the ground station. As clearly seen in the map, there exists large scale structure from Galactic cirrus emission; with the central region darker than the surrounding area. Most of the bright spots can be attributed to nearby galaxies.

\subsection{Stars and galaxies}

Each star or galaxy in the field does not contribute much to the overall sky brightness, but the total contribution including faint sources down to the detection limit is non-negligible for accurate measurements of the CIB. Moreover, point sources seriously affect the sky fluctuations rather more than the absolute brightness. Hence, we masked out foreground stars and galaxies down to the $3 \sigma$ detection limit of $\sim 100 \mathrm{mJy}$ at $65 \mu \mathrm{m}$ and $\sim 20$ mJy at $90 \mu \mathrm{m}$, from the diffuse map. The mask is a circular area around each source with the radius normalized to be 1 arcmin at $100 \mathrm{mJy}$ increasing proportionally with increasing flux. The lower image of Figure 4 is the resultant $90 \mu \mathrm{m}$ map after the source removal. The masked areas are filled with zero values to make the source locations clear.

According to the point spread function (PSF) previously measured for stars and asteroids as calibration standards (Shirahata et al. 2009), the remaining brightness in the out-of-mask region around a point source is less than $5 \%$ of the peak brightness, and the integrated flux in the out-ofmask region is $20 \%$ of the total flux. The integrated flux of the out-of-mask region of all sources down to the $3 \sigma$ detection limit is less than half of the total source flux. Consequently, the mean brightness of zodi-subtracted map after removing the sources in such way is $\sim 3 \%$ lower than the original value including the sources, and the sky fluctuation power is also an order of magnitude lower. We have also confirmed that the fluctuation power has the same value even if we change the mask aperture by a factor of $\pm 50 \%$. This implies the contribution of the out-of-mask fringes of PSF to the total sky fluctuations is negligible.

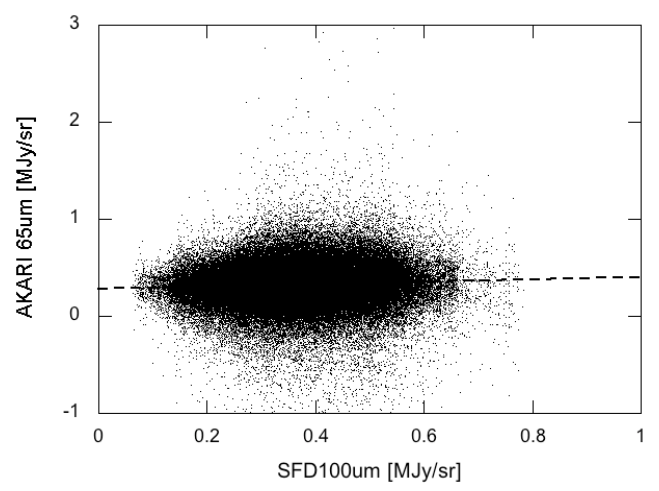

Fig. 5.- Correlation between the AKARI $65 \mu \mathrm{m}$ map and the SFD $100 \mu \mathrm{m}$ map. The dashed line is the best fit to the data.

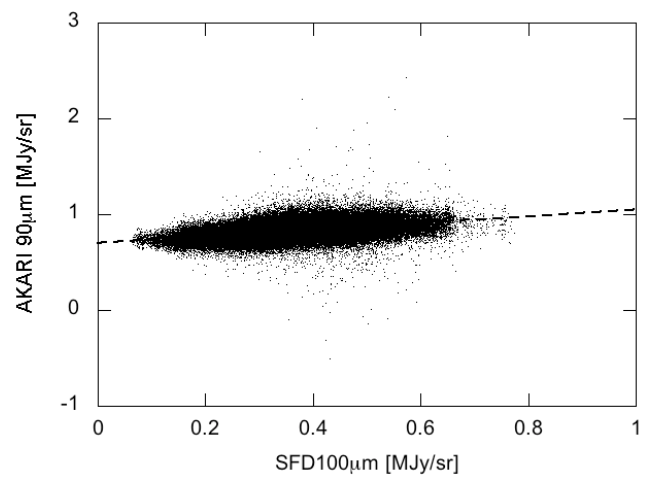

Fig. 6. - Correlation between the AKARI $90 \mu \mathrm{m}$ map and the SFD $100 \mu \mathrm{m}$ map, same as Figure 5

\subsection{Galactic cirrus}

To estimate the Galactic foreground, we studied the correlation between the ADF-S map and 
the SFD dust map (Schlegel et al. 1998), which is widely used as a reliable map of Galactic cirrus. This correlation may be scattered by point sources since the angular resolution of AKARI is much higher than that of the SFD map based on the IRAS and COBE data. In fact, many bright galaxies in the field are resolved by AKARI, but in the SFD map they are left unresolved and recognized as part of the cirrus structure instead. Thus, we calculated the correlation for the zodi-subtracted map after removing bright galaxies and reducing the map resolution to be the same as SFD.

Figure 5 and Figure 6 show the correlation between the SFD map at $100 \mu \mathrm{m}$ and the ADF-S map at 65 and $90 \mu \mathrm{m}$, respectively. The $90 \mu \mathrm{m}$ map correlates significantly with the SFD map with a correlation coefficient of $r=0.43$, and the linear coefficient from the best fit line, shown in Figure 6 by the dashed line, is $I(90 \mu \mathrm{m}) / I(100 \mu \mathrm{m})=0.7030 \pm 0.0007$. This color ratio corresponds to a dust temperature of $T=18.2$ $\mathrm{K}$ for $n=2$, where $n$ is the emissivity index and $I(\nu) \sim B(T) * \nu^{-n}$, and is consistent with typical dust properties (Schlegel et al. 1998). The correlation for the $65 \mu \mathrm{m}$ map was marginally seen as $r=0.05$ with $I(65 \mu \mathrm{m}) / I(100 \mu \mathrm{m})=0.283 \pm 0.002$.

It is remarkable that the zero intercepts of the best-fit lines for the correlations for both the 65 and $90 \mu \mathrm{m}$ data show significantly finite values, which implies a positive detection of an isotropic emission component of the CIB. To estimate the cirrus brightness in the long wavelength bands, we extrapolated the $90 \mu \mathrm{m}$ brightness with a blackbody spectrum of $T=18.2 \mathrm{~K}$ for $n=2$, because the map quality was not high enough to allow the same correlation analysis as made for the short wavelength bands.

\section{Power spectrum analysis}

\subsection{Method}

In order to carry out a fluctuation study of CIB, we have made a power spectrum analysis using the two-dimensional (2-D) FFT for the final zodisubtracted maps of the ADF-S. For the 2-D FFT analysis, we first produced a co-added image of the entire field with Cartesian coordinates by a simple tangential projection. The image size is so small that the distortion by the projection does not affect the result (while a spherical harmonics expansion with multipoles is required for power spectrum analysis at angular scales larger than 10 deg). Next, we subtracted the mean offset level from the image, we set the blank data area out of the boundary of the observed area (dark area in Figure 4) to zero value, and we also set the sourcemasked regions to zero value. Then, we applied a 2-D FFT to the entire image. Finally, we corrected the raw power spectrum in the spectral domain by correction factors such as effective area, noise spectrum and PSF, as described below.

\subsection{Effective area}

As seen in Figure 4, the survey field has a nonrectangular shape, and the image includes zerovalue data. Such an irregular shape may distort the power spectrum, and the non-negligible number of zero-value data points decreases the amplitude of the power spectrum. In order to obtain the real power spectrum avoiding such artifacts, we have corrected the power spectrum by multiplying it by a correction factor corresponding to the effective area of the field.

To derive this correction factor, we used a random noise simulation method by generating noise images of the same shape and area as the survey field, so that the root-mean-square noise of all the images have a constant value. Then, we calculated the power spectra of the noise images by using the 2-D FFT. Since the simulated noise image should have a flat power spectrum, i.e., white noise, we can find any effects due to image irregularity as a change in distortion and amplitude of the noise power spectra. The correction factor was actually calculated as the ratio between the original white noise spectrum and the mean power spectrum of the simulated noise images.

\subsection{Angular power spectrum}

Figure 7 shows the power spectrum of the 90 $\mu \mathrm{m}$ map including bright galaxies as a function of angular wavenumber. The amplitude is corrected by the effective area as described above. To obtain the one-dimensional power spectrum, we have projected the 2-D power spectrum onto a radial axis of polar coordinates in angular wavenumber by calculating the ensemble mean for phase angles in a coaxial ring of each bin of the radial axis. The bin width is approximately $0.003 \operatorname{arcmin}^{-1}$ corre- 


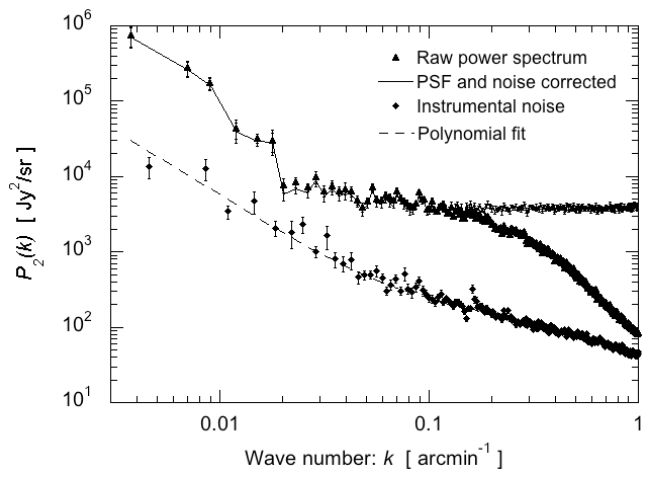

Fig. 7.- Raw power spectrum of the zodisubtracted ADF-S map at $90 \mu \mathrm{m}$ including bright sources (filled triangles), and the PSF corrected power spectrum after subtraction of the instrumental noise (thin line). The diamonds represent the measured power spectrum of the instrumental noise, and the dotted line is the result of a polynomial fit to the noise spectrum.

sponding to the inverse of the angular size of the map. The error bars of the power spectrum denote the standard error calculated from the standard deviation and number of data in each radial bin.

\subsection{Noise estimate}

In order to obtain the fluctuation power with astronomical origin, the subtraction of the instrumental noise is necessary. We estimated the noise power spectrum from the difference of two subset maps taken in the same field but in different seasons. The obtained noise power spectrum shown in Figure 7 was dominated by the low-frequency noise as fitted by polynomials with terms of $1 / f$ and $1 / f^{2}$ noise. For the noise subtraction we used the result of polynomial fit rather than the data points to avoid adding any artifacts.

The estimated noise is mainly due to instrumental noise but may include systematic errors of the zodi-model uncertainty and the responsivity correction. The seasonal variation of small-scale structures of the zodiacal emission may also contribute to the noise, but its contribution is also subtracted from the measured power spectrum as a noise component. This noise level gives an upper limit for the residual fluctuations of zodiacal emission smaller than $0.5 \%$ of the mean level at angular scales of $\sim 1$ arcmin, while the previously reported upper limit at the same angular scale is 1\% (Abraham et al. 1998).

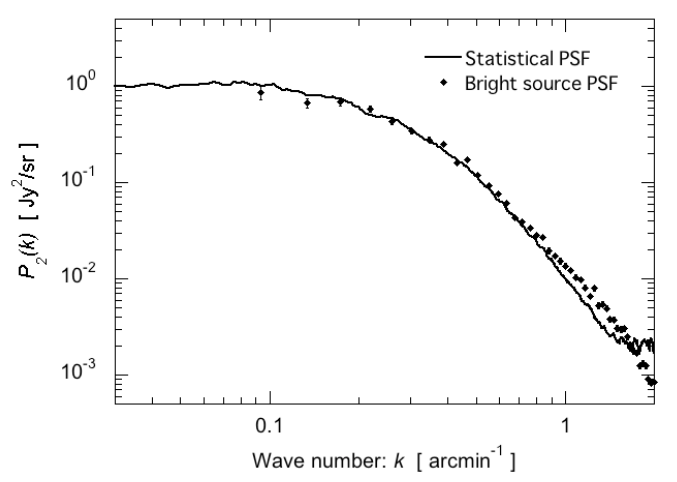

Fig. 8. - The normalized power spectrum of PSF at $90 \mu \mathrm{m}$ directly obtained from the ADF-S map itself by two methods. The thin line is the statistical PSF obtained by differentiating the maps before and after removing point sources. The data points represent the average PSF for bright sources in the field.

\subsection{Beam correction}

The steep decrease of the fluctuation power towards large wave numbers is obviously due to low-pass filtering by the PSF, which has a beam size of approximately 30 arcsec in FWHM. In order to reproduce the intrinsic power spectrum of the map, we divided the raw power spectrum by the normalized power spectrum of the PSF constructed from sources in the ADF-S field itself (self-measured PSF). Although the pre-measured PSF of bright stars as calibration standards could also be used for this purpose, observations of calibration stars with the same AOT parameter as the ADF-S survey were not available because the calibration sources are too bright.

Figure 8 shows two kinds of power spectra for the self-measured PSF; one is a statistical PSF spectrum from the difference between two spectra of the source-removed map and the original map, and the other is the average PSF spectrum of bright sources only in the field. To obtain the average PSF spectrum, we cut out postage stamps with a size of $40 \times 40 \operatorname{arcmin}^{2}$ around the bright 
sources with signal-to-noise ratios higher than 30 . The statistical PSF spectrum is normalized at the small end of wavenumber, and the average PSF spectrum is scaled to fit the overall range. The 2 methods show good agreement with each other in the range of 0.1-0.8 arcmin, though a large discrepancy appears at large wave numbers. Since this paper focuses on the power spectrum at large angular scales we have adopted the statistical PSF, because it actually provides a better result in order to reproduce the power spectrum of the map at large wavenumbers and it is also known that transient effects are stronger for brighter sources (Shirahata et al. 2009).

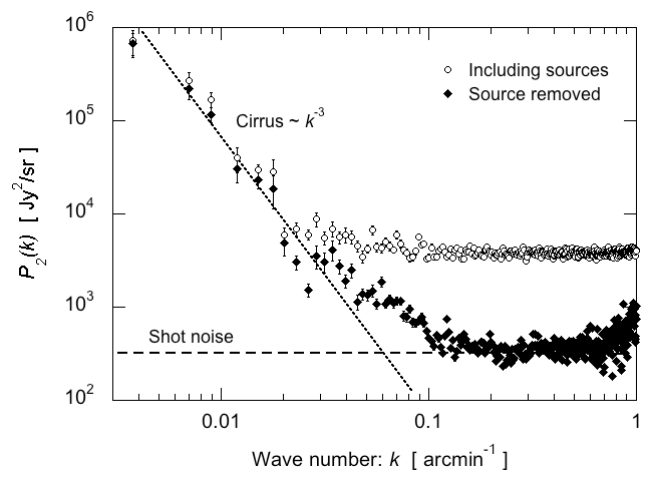

Fig. 9.- Power spectrum of the ADF-S map after removing point sources (filled diamonds) compared with the power spectrum including the sources (open circles). The dotted line is the third power law for Galactic cirrus. The dashed line represents the shot noise level due to individual contribution of unresolved galaxies.

\subsection{Resultant power spectrum}

\subsubsection{Raw power spectrum}

The power spectrum after the noise subtraction and the PSF correction is shown in Figure 7. There exist two major components in the power spectrum. One is the constant component dominating at wavenumbers larger than $0.1 \operatorname{arcmin}^{-1}$, and another shows a steep rise towards the smallest wave number following a $k^{3}$-power law. The former is regarded as the shot noise (Poisson noise) mainly due to bright galaxies in the field, and the latter can be identified as Galactic cirrus fluctua- tions, as discussed in the following section.

We have carried out a power spectrum analysis for the $65 \mu \mathrm{m}$ data in the same way as the $90 \mu \mathrm{m}$ data. However, the obtained power spectrum was not significant compared to the noise power, suffering from low sensitivity. It was also difficult to obtain a significant power spectrum at 140 and 160 $\mu \mathrm{m}$ because of the presence of low-frequency noise originating from the responsivity change induced by cosmic-ray hits.

\subsubsection{Bright source removal}

The raw power spectrum in Figure 7 is obviously contaminated by shot noise with a flat spectrum at larger wavenumbers, originating from randomly distributed bright galaxies in the field. To investigate the fluctuations originating from underlying faint galaxies (the building blocks of the CIB), we derived the power spectrum removing the point sources down to the detection limit of AKARI, 20 mJy.

Figure 9 shows the PSF-corrected power spectra before and after the point source removal. The shot noise power, in contrast to the cirrus component, decreases to $10 \%$ of the value before the source removal. The increased power at $\sim 1$ $\operatorname{arcmin}^{-1}$ may be the signature of small-scale correlation between galaxies and source confusion or may be due to the uncertainty of the PSF as seen in Figure 8. Study of the power spectrum at these small angular scales is reserved for future work. At the other wavelengths, we have only obtained upper limits for the shot noise, which are not so meaningful as new limits.

\section{Results and Discussion}

\subsection{Mean CIB levels}

\subsubsection{Absolute brightness towards the ADF-S}

The resultant CIB brightness was measured as the zero-intercept of the correlation between the zodi-subtracted map and the SFD map as shown in Figure 10 and summarized in Table 2 with statistical and calibration errors with an uncertainty in the zodiacal emission model of $5 \%$ (Kelsall et al. 1998). In Table 2 the mean brightness of zodiacal emission and Galactic cirrus emission over the entire ADF-S field are also shown as components of the measured total sky brightness. The measured 
brightnesses in Table 2 are all color-corrected assuming a flat spectrum. Although the zero-point offset of the SFD map may cause a systematic uncertainty in the CIB brightness, such uncertainty has been estimated to be smaller than 0.1 MJy $\mathrm{sr}^{-1}$ at $100 \mu \mathrm{m}$ (Finkbeiner et al. 2000), which does not significantly alter the results. In conclusion we can say we have significantly detected the CIB in the short wavelength bands. We have also tentatively detected the CIB at 140 and $160 \mu \mathrm{m}$ by extrapolating the cirrus brightness from $90 \mu \mathrm{m}$ to each wavelength assuming a $T=18.2 \mathrm{~K}$ blackbody spectrum.

In order to obtain a more robust result, if we adopt another zodi-model with a very powerful assumption about the celestial signal called "verystrong no-zodi" principle, i.e., no extrasolar emission at $25 \mu \mathrm{m}$ exists at high ecliptic latitudes (Wright et al. 1998; Wright 2001; Levenson et al. 2007), then the zodi-model brightness becomes $\sim 10 \%$ higher than what was assumed for our foreground subtraction (L. Levenson 2009, private communication). On this assumption, the residual background decreases completely to zero at $65 \mu \mathrm{m}$ but still remains at $90 \mu \mathrm{m}$ at a significant positive value of $0.50 \mathrm{MJy} \mathrm{sr}^{-1}$, because of the bluer color of zodiacal emission. This lower limit of the CIB at $90 \mu \mathrm{m}$ is shown in Figure 10. In the long wavelength bands, the zodi-model difference changes the residual background by at most $10 \%$, which is smaller than the statistical error.

\subsubsection{Isotropy}

In Table 3, we summarize the zodi-subtracted sky brightness in various fields as a mean value with the error estimated from the brightness variance for a number of observations in each field. The error does not include either the calibration error or the zodi-model uncertainty. The table also indicates the residual brightness after subtracting the cirrus brightness, which were estimated from the HI $20 \mathrm{~cm}$ line map by the LeidenArgentine-Bonn (LAB) survey (Kalberla et al. 2005). The cirrus brightness was obtained by assuming a conversion factor from the HI-column density to the $100 \mu \mathrm{m}$ brightness of, $0.6 \pm 0.1 \mathrm{MJy}$ $\mathrm{sr}^{-1} / 10^{20} \mathrm{~cm}^{-2}$, as the median value of various COBE-based results with their variation as an uncertainty (Boulanger et al. 1996; Reach et al. 1998; Schlegel et al. 1998; Lagache et al. 2000a).

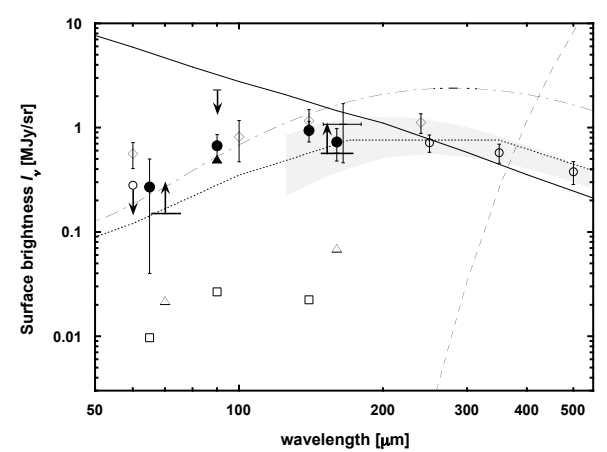

Fig. 10. - The CIB brightness measured in the ADF-S (filled circles), compared with the previous CIB limits from COBE/DIRBE (diamonds), COBE/FIRAS (shaded region), IRAS (open circle with downward arrow at $60 \mu \mathrm{m}$ ), ISO (thin line with downward arrow at $90 \mu \mathrm{m}$ and cross in 150$180 \mu \mathrm{m}$ ), Spitzer (upward arrows) and BLAST (open circles at 250,350 and $500 \mu \mathrm{m}$ ). The filled triangle at $90 \mu \mathrm{m}$ is the lower limit of the CIB derived for the case of "very-strong no-zodi" principle (Wright 2001; Levenson et al. 2007). The solid line shows a typical spectrum of the zodiacal foreground at the ecliptic poles. The dashed line is the CMB. Model predictions from Lagache et al. (2004) and Takeuchi \& Ishii (2010) are indicated by the dotted and dot-dashed curves, respectively. The open triangles and the open squares are lower limits obtained from the integrated flux of the galaxy counts with Spitzer and AKARI, respectively.

For the wavelength conversion from $100 \mu \mathrm{m}$ to the AKARI bands, we used the correlation coefficients in Figure 5] and Figure 6. Since the angular resolution of the HI data is 10 arcmin, much larger than that of our data, we used the mean brightness of our data and of the HI data in each field. The residual brightness after subtraction of the cirrus brightness in different fields agree with each other within the error bars at both 65 and $90 \mu \mathrm{m}$.

The ratio of the HI-column density to the farinfrared brightness assumed above may be an overestimate, because it tends to be determined by the data in the brighter cirrus regions. In low cirrus regions the ratio could be smaller due to the contribution of uncorrelated components, such as 
dust associated with ionized gas. In fact, the mean residuals for the three fields in Table 3 are slightly smaller than the results in Table 2 from the correlation of the ADF-S and SFD maps, though they are consistent with each other within the total error including the calibration error. The contribution of cirrus could be more accurately estimated in the long wavelength bands, where the cirrus emission is brighter. However, we have not made the same analysis as the short wavelength bands, because accurate measurement of CIB was possible only in the ADF-S field which has highly statistical samples.

\subsubsection{Comparison with previous observations}

As seen in Figure 10, our results in the four photometric bands show good agreement with previous CIB measurements from COBE at 60, 100 and $140 \mu \mathrm{m}$ (Hauser et al. 1998). They are also consistent with an upper limit from IRAS at $60 \mu \mathrm{m}$ (Miville-Deschênes et al. 2002) and with an upper limit at $90 \mu \mathrm{m}$ and the measured CIB level in the $150-180 \mu \mathrm{m}$ range measured by ISO (Juvela et al. 2009).

The integrated galaxy counts (Dole et al. 2004; Matsuura et al. 2007; Shirahata et al. 2008) account for only a few per cent of the CIB brightness. Stacking analysis of the Spitzer $24 \mu \mathrm{m}$ sources (Dole et al. 2006) substantially extended the lower limits at 70 and $160 \mu \mathrm{m}$ to the prediction from the galaxy evolution model presented in Lagache et al. (2004). Our measured CIB brightness at $90 \mu \mathrm{m}$ is obviously higher than the Spitzer result, and our result implies the existence of large number of unknown sources, which may have been missed in the stacking process since they were too faint to be detected at $24 \mu \mathrm{m}$ with Spitzer.

A hypothetical source with such an excess brightness should have peculiar spectra with a peak around $100 \mu \mathrm{m}$, so that they have only a small contribution to the CIB in the other wave bands. High levels of the CIB at $100 \mu \mathrm{m}$ have already been seen with COBE (Hauser et al. 1998; Finkbeiner et al. 2000) but have been thought to be due to calibration errors and foreground uncertainties (Dole et al. 2006). It is remarkable that this work corroborates with the COBE result using a different instrument.

\subsubsection{Possible origins of the excess emission}

If the excess emission at $90 \mu \mathrm{m}$ originates from an isotropic component of the Galactic cirrus, which may not be accounted for in the SFD map, it should be tightly constrained by the previously measured HI-column density unless the gas-todust ratio is extraordinary. If the excess brightness at $90 \mu \mathrm{m}$ of $\sim 0.4 \mathrm{MJy} \mathrm{sr}^{-1}$ is associated with neutral Hydrogen, the corresponding HI-column density must be $N_{H I} \sim 1 \times 10^{20} \mathrm{~cm}^{-2}$. This is much higher than the measured HI-column density towards the ADF-S (see Table 3) and unlikely, judging from uncertainty of the $\mathrm{HI}$ observation. Moreover, if the temperature of such isotropic dust emission is $\sim 17 \mathrm{~K}$, similar to the bulk of the Galactic cirrus emission, it should appear at longer wavelengths as a strong excess, but this is not significant in our data. Therefore, it is difficult to explain the excess brightness by dust emission associated with neutral Hydrogen.

Unexpectedly large amounts of ionized gas at high galactic latitudes as a warm ionized medium (WIM) is a another possible candidate for the excess emission. In fact, dust emission from a WIM has been detected with the WHAM $\mathrm{H}_{\alpha}$ survey, though the observed areas have been limited to relatively dense regions at low galactic latitudes (Lagache et al. 2000a). Large differences in the residual brightness between different fields shown in Table 3 can be caused by the contribution of dust associated with a WIM. In fact, the ADF-S is darker than the LH in the far-infrared, while the HI-column density towards ADF-S is higher than that towards LH. However, any WIM could be dust poor compared to the Galactic plane, because grain destruction is expected to occur in low density regions of the interstellar medium, while the ionized Hydrogen column density to account for all of the excess brightness must be higher than that of the neutral Hydrogen. As in the case of the neutral Hydrogen, the dust temperature must be so high as to have an emission peak around $100 \mu \mathrm{m}$ while not producing a significant excess at longer wavelengths, but the existence of such hot dust at high galactic latitudes has not been reported. Future measurements of the $\mathrm{H}_{\alpha}$ emission and other interstellar lines in the ADF-S would be important tests of the WIM hypothesis to explain the excess.

Star-forming galaxies at high redshift are an- 
other possible origin of the CIB excess in the farinfrared. In Table 2. the measured CIB brightness is compared with the range predicted from a selection of galaxy evolution models, which take the contribution of a population of luminous infrared galaxies peaking at $z \sim 1$ and ultra-luminous infrared galaxies at $z>2$ into consideration (Lagache et al. 2004; Pearson et al. 2007; Franceschini et al. 2008; Takeuchi \& Ishii 2010). Most of these galaxy evolution models give a lower brightness than our measured CIB at 65 and $90 \mu \mathrm{m}$, while the models agree with our result at 140 and $160 \mu \mathrm{m}$, the stacking analysis of Spitzer data at 70 and $160 \mu \mathrm{m}$, and the COBE results at wavelengths other than $100 \mu \mathrm{m}$, within the data uncertainties. A model prediction from Takeuchi \& Ishii (2010) in Figure 10 shows a fairly good agreement with the measured CIB at wavelengths shorter than $140 \mu \mathrm{m}$, but it gives too high brightness at longer wavelengths, especially in the submillimeter range (Puget et al. 1996; Fixsen et al. 1998). It is obvious that our result especially at $90 \mu \mathrm{m}$ provides a tight constraint on galaxy evolution models.

In contrast to the CIB measurement, the source counts at $90 \mu \mathrm{m}$ with AKARI were much lower than those predicted from contemporary galaxy evolution models, while the galaxy counts at 65 and $140 \mu \mathrm{m}$ are consistent with the models and the Spitzer results at 70 and $160 \mu \mathrm{m}$ (Matsuura et al. 2007; Shirahata et al. 2008). Such low counts at $90 \mu \mathrm{m}$ have also been found with ISO (Rodighiero et al. 2003; Heraudeau et al. 2004), and it has been reported that many galaxy evolution models fail to reproduce the $90 \mu \mathrm{m}$ counts (Chary \& Elbaz 2001; Serieant et al. 2004). At $90 \mu \mathrm{m}$ the integrated galaxy counts down to the detection limit of AKARI, $\sim 20 \mathrm{mJy}$, can only account for less than $10 \%$ of the CIB brightness (Matsuura et al. 2007). To reproduce the CIB brightness, a strong increase in the galaxy counts below the detection limit is necessary. If we assume a single power law with power index of -1.5 (Euclidian) for the cumulative galaxy counts, $d N / d S \sim S^{-1.5}$, the power law needs to extend to $0.01 \mathrm{mJy}$. Such high counts require an extraordinary evolution scenario because conventional galaxy evolution models predict a steep drop of the differential $90 \mu \mathrm{m}$ counts towards the faint end below $1 \mathrm{mJy}$.
To explain the previous COBE result of high CIB levels at 60 and $100 \mu \mathrm{m}$, the possible contribution of a new population at high redshift $(z=2-3)$ with high dust temperatures $\left(T_{d} \sim 60\right.$ $\mathrm{K})$ heated by AGN, or accretion onto a black hole, has already been discussed (Finkbeiner et al. 2000; Blain \& Phillips 2002; Blain et al. 2004). If this is true, our finding of the CIB excess at 90 $\mu \mathrm{m}$ could also be explained by such high- $z$ hot sources. Many results from the Spitzer $24 \mu \mathrm{m}$ and $\mathrm{X}$-ray surveys suggest that LIRG and ULIRG at $z \sim 1$ rather than AGN at high redshift constitute main bulk of the CIB, and the contribution of AGN to the CIB is thought to be at most $10 \%$ (Treister et al. 2006; Devlin et al. 2009). Therefore, the CIB excess is more likely to be composed of high- $z$ hot sources of new galaxy population rather than known obscured AGN, which could be detected at $24 \mu \mathrm{m}$. If the hot source is so heavily obscured that the dust extinction is strong even in the mid-infrared, and if the dust grains are so small that the emissivity index is a maximum, $\lambda^{-2}$, at longer wavelengths, they can contribute to the CIB selectively at short far-infrared wavelengths.

\subsubsection{Energetics and cosmological implications}

The total integrated energy of the CIB excess in a range of the AKARI bands is estimated to $\sim 10 \mathrm{nWm}^{2} \mathrm{sr}^{-1}$, which is comparable to the CIB energy associated with the $24 \mu \mathrm{m}$ galaxies of 24 $\mathrm{nWm}^{2} \mathrm{sr}^{-1}$ (Dole et al. 2006). If we assume a constant star formation rate (SFR) over cosmic history of $z>1$, the excess energy corresponds to the SFR of $\sim 0.1 \mathrm{M}_{\odot} \mathrm{yr}^{-1} \mathrm{Mpc}^{-3}$, which is an order of magnitude higher than that in local universe (Hauser \& Dwek 2001). If the radiation energy is produced by a star burst at $z$, the total hydrogen mass fraction converted to metals in this epoch is estimated to $\Delta X \sim 0.004(1+z)$. For redshift of $z>1$, more than $1 \%$ of baryon mass density needs to be converted to metals. Starburst at high- $z$ as the origin of the CIB excess may cause over production of metals beyond the metal abundance in local universe. Black hole accretion may pray important roll to generate the background radiation at high redshift (Finkbeiner et al. 2000).

In the near-infrared, the CIB excess which cannot be explained by the integrated flux of known galaxy populations has been reported, and the ori- 
gin of the excess as first stars, or proto-galaxies, at $z \sim 10$ has been discussed in terms of the spectral signature of the redshifted $\mathrm{Ly}-\alpha$ in the CIB near $1 \mu \mathrm{m}$, as seen in Figure 1 (Kashlinsky 2005; Matsumoto et al. 2005). Findings of the CIB excess in both near- and far-infrared lead us to consider their connection; the UV light from the first massive stars which produce the near-infrared excess might be partly absorbed by the self-produced dust, re-emitted in the mid-infrared and redshifted into the far-infrared band at present. Accretion to the first blackhole as a remnant of the first star explosion might also be the hot source at high redshift. Dust production by the first stars and successive process as blackhole formation and accretion is little understood, and this research subject would be proceeded by future observations of infrared galaxies and the CIB in many details.

\subsection{Fluctuations of the CIB}

\subsubsection{Galactic cirrus}

In order to obtain information on the CIB fluctuations from the measured power spectrum, it is necessary to estimate the fluctuation power of the foreground such as the zodiacal emission and the Galactic cirrus. Zodiacal emission, the most dominant foreground component, has been known to have a very smooth distribution, and its fluctuations in the mid-infrared have been measured with ISO to be smaller than $1 \%$ of the mean brightness at angular scales of 1 arcmin (Abraham et al. 1998). This value is already much smaller than the shot noise level by unresolved galaxies. Although an unexpected time varying residual of zodiacal emission after the zodi-model subtraction may contribute to the power spectrum over a broad range of angular scales, it has already been subtracted as a part of the noise power estimated from the difference of the two sub-maps, as described in the last section. Thus, the main foreground is Galactic cirrus, especially at large angular scales.

A $k^{3}$-power law of the fluctuation spectrum for Galactic cirrus has been reported by many authors, e.g., Miville-Deschênes et al. (2007), but it is remarkable that in this work the same power law was confirmed at such very low flux levels at $90 \mu \mathrm{m}$. The power spectrum of the cirrus fluctua- tions can be expressed as

$$
P_{\text {cirrus }}(k)=P_{0}\left(k / k_{0}\right)^{-\gamma},
$$

where $k$ is the angular wavenumber in $\operatorname{arcmin}^{-1}$, and $P_{0}$ is the power normalized at $k_{0}=0.01$ $\operatorname{arcmin}^{-1}$. $\gamma$ is the power law index, ranging from 2.5 to 3.1 and is known to be close to 3 for the optically thin case at low cirrus regions (Schlegel et al. 1998; Jeong et al. 2005; Miville-Deschênes et al. 2007). As shown in Table 4, our result gives the cirrus power at $90 \mu \mathrm{m}$ of $P_{0}=(5.5 \pm 0.5) \times 10^{4}$ $\mathrm{Jy}^{2} \mathrm{sr}^{-1}$, and the power at $100 \mu \mathrm{m}$ is derived to be $P_{0}=(1.1 \pm 0.1) \times 10^{5} \mathrm{Jy}^{2} \mathrm{sr}^{-1}$ from the correlation coefficient between the ADF-S and SFD maps.

At high values of $k$ the power law index may not always be the same as the value derived at low $k$, where the cirrus component dominates the total fluctuation power. In previous work with the ISO observations, it has been proved that a single power law for the cirrus power spectrum is valid up to $k \sim 0.3 \operatorname{arcmin}^{-1}$, though this result is certain only in relatively bright regions. Since there is no strong reason to change the power law index at higher $k$, we assume a single power law over the entire wavenumber range for the estimate of the cirrus power.

It has been reported that the fluctuation power of the cirrus emission scales with the mean brightness as a power law with index $2-3$ depending on the brightness. According to Miville-Deschênes et al. (2007), the cirrus fluctuation power at $100 \mu \mathrm{m}$ in the low brightness regime $\left(B<10 \mathrm{MJy} \mathrm{sr}^{-1}\right)$ is given by

$$
P_{0}=2.7 \times 10^{6} B^{2.0} J^{2} s r^{-1},
$$

where $B$ is the mean brightness at $100 \mu \mathrm{m}$. If this is the case, our measured power of the cirrus fluctuations corresponds to the mean brightness at 90 $\mu \mathrm{m}$ of $0.14 \mathrm{MJy} \mathrm{sr}^{-1}$, which is slightly lower than the value in Table 2. The relation between the fluctuation power and the mean brightness may not be valid under such low cirrus conditions. The result implies that it is dangerous to estimate the mean CIB brightness from the fluctuation power.

\subsubsection{Shot noise of unresolved galaxies}

We have derived the shot noise of unresolved galaxies from the source -removed power spectrum in Figure 9 as the mean power level in a 
range of $0.2-0.8 \operatorname{arcmin}^{-1}$, where the PSF is relatively accurate, to be $P_{\text {shot }}=(3.6 \pm 0.2) \times 10^{2} \mathrm{Jy}^{2}$ $\mathrm{sr}^{-1}$ at $90 \mu \mathrm{m}$. In Table 4 we summarize our result for the foreground estimates at small angular scales, where the shot noise dominates, into known emission components. The fluctuations of zodiacal emission was estimated to be negligible from the discussion in the last section. The cirrus component following a $k^{3}$-power law is negligible at such small angular scales $\left(k>0.2 \operatorname{arcmin}^{-1}\right)$. Therefore, the remaining shot noise can be attributed to the fluctuations from unresolved galaxies.

In Table 4 we compare our result with the previous work at $90 \mu \mathrm{m}$ with ISOPHOT (Matsuhara et al. 2000) and with a range of the shot noise levels predicted from various galaxy evolution models. Since the detection limit of AKARI for point sources is better than that of ISO, the AKARI result shows obviously lower fluctuation power than the ISO result. We have estimated the shot noise level for each galaxy evolution model by simply integrating the squared flux, $S^{2}$, with the differential source counts, $d N / d S$, as

$$
P_{\text {shot }}=\int S^{2}(d N / d S) d S
$$

for fluxes below $20 \mathrm{mJy}$. The measured shot noise power is 3-5 times lower than any of the model predictions clearly due to the high source counts of the models near the detection limit. The result implies that the main bulk of the CIB at $90 \mu \mathrm{m}$ consists of sources much fainter than the detection limit.

Since the shot noise is the squared flux integrated with the source counts, it is more sensitive to the number density of bright sources than the mean CIB level, that is a linear integration of the flux. As described in the last section, the mean CIB level provides constraints on the source counts at the very faint end below the point-source detection limit. In contrast, the shot noise can provide constraints on the source counts near the detection limit.

The measured shot noise level can be reproduced by the measured source counts extended down to $1 \mathrm{mJy}$ with a Euclidian power law (a constant for the differential counts). In such a simple scenario, sources fainter than $1 \mathrm{mJy}$ have little contribution to the fluctuations but become the main contributor to the mean brightness. In order to resolve the bulk of the CIB into point sources, future infrared telescope with the detection limits much better than $1 \mathrm{mJy}$ at $\sim 100 \mu \mathrm{m}$ would be required.

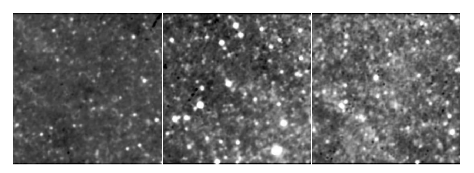

Fig. 11. - Sub-maps cut out from the selected positions in the ADF-S map at $90 \mu \mathrm{m}$; left: center region with lowest brightness, mid: a cluster of galaxies (Abell s0463), and right: relatively high cirrus region. The image sizes for all maps are the same, $1 \times 1 \mathrm{deg}^{2}$.

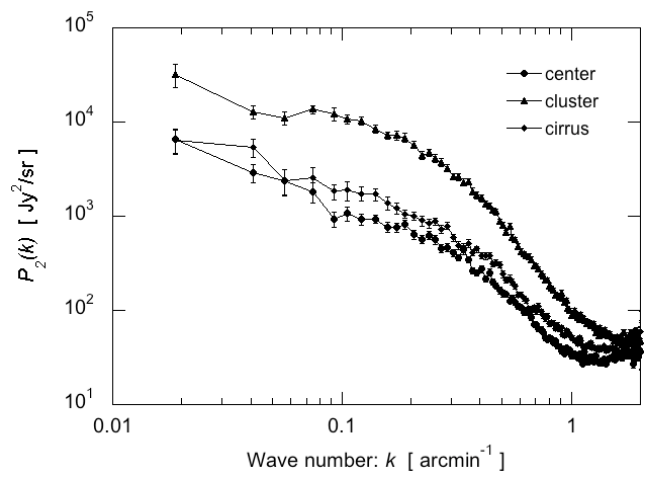

Fig. 12.- Power spectra of the three maps in Figure 11. Circles, triangles and diamonds represent data points for the central, the cluster of galaxies, and the high cirrus regions, respectively. These power spectra are raw data without noise subtraction and the PSF correction.

\subsubsection{Cosmic variance}

Since the ADF-S covers a large contiguous area of 12 sq.deg, the field contains various regions of 


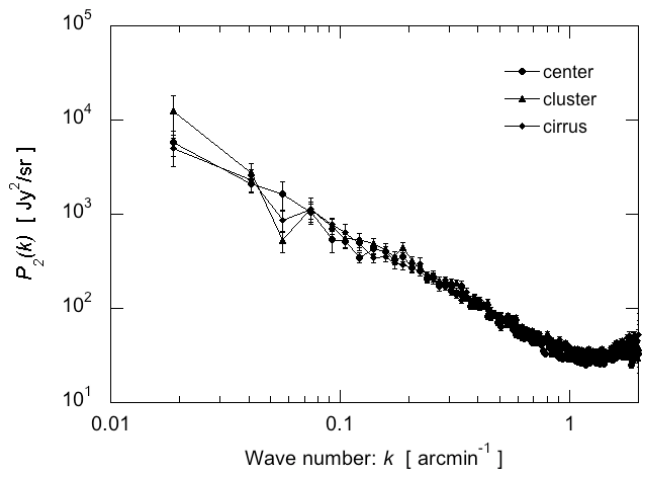

Fig. 13. - Power spectra for the sub-maps same as in Figure 12 after the point source removal.

different source and cirrus densities. The power spectrum shown in Figure 9 gives the average properties of the entire field, but it can be biased due to strong features of specific regions in the map.

In order to check for any cosmic variance, we have carried out power spectrum analysis for sub maps of $1 \times 1 \mathrm{deg}^{2}$ area cut out from various positions as shown in Figure 11. One is the central region with the minimum cirrus level and source densities, the second region contains a rich cluster of galaxies DC 0428-53 (Abell S0463) (Dressler 1980), and the third is a relatively high cirrus region with a moderate source density. As shown in Figure 12, the power spectrum obtained for the cluster region is about an order of magnitude higher than the others. The central region shows the minimum fluctuation power as expected, and the high cirrus region has slightly higher power than the central region, though the point source contribution seems to be more prominant rather than the cirrus fluctuations. This result implies that the power spectrum is severely affected by the point source contribution and biased to high source density regions.

In order to estimate the effect of point sources on the power spectrum in detail, we have made the same comparison of the three sub maps after removing point sources. The result is shown in Figure 13. All of the power spectra at the different sky positions show excellent agreement with each other at an accuracy better than 5\% in the overall range of angular wavenumber. This result implies that the power spectrum after removing point sources is not affected by cosmic variance such as the clustering of nearby galaxies and can be regarded as a universal function for the CIB fluctuations.

\subsubsection{Clustering component}

In the measured power spectrum in Figure 9, a fluctuation component of $\sim 10^{3} \mathrm{Jy}^{2} \mathrm{sr}^{-1}$ is seen in the range of $0.03-0.1 \operatorname{arcmin}^{-1}$. Since the $k$ dependence of this component is moderate and apparently less steep than the $k^{3}$-power law, it is not likely to be attributed to either shot noise of unresolved galaxies or Galactic cirrus. No excess fluctuations of Galactic cirrus on such a small scale have been found in the high cirrus density region, where the cirrus dominates the sky fluctuations.

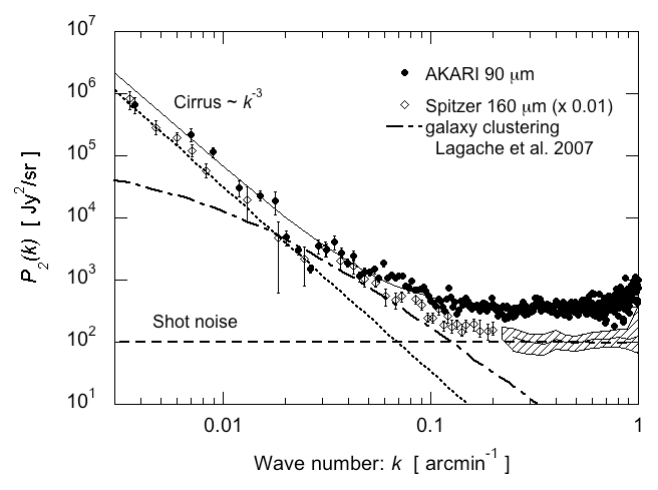

Fig. 14. - The final power spectrum at 90 $\mu \mathrm{m}$ (filled circles), same as in Figure 9, is compared with the Spitzer/MIPS $160 \mu \mathrm{m}$ result in Lagache et al. (2007) (open diamonds and a shaded region at $\left.k>0.2 \operatorname{arcmin}^{-1}\right)$ and their model of galaxy clustering (dash-dotted curve), Galactic cirrus (dotted curve) and shot noise (dashed line). The Spitzer result is multiplied by a factor of 0.01 to adjust the vertical scale. The solid thin line is the best fit to the AKARI data with a multiple power-law model (see text).

A plausible interpretation of the fluctuation power at intermediate angular scale is the clustering of star-forming galaxies, e.g., Perrotta et al. (2003). Lagache et al. (2007) have reported their finding of galaxy clustering in the power spectrum at $160 \mu \mathrm{m}$ with Spitzer after removing point 
sources brighter than 200 mJy. In Figure 14, we plot the Spitzer result from Lagache et al. (2007) multiplied by a scaling factor of 0.01 with our power spectrum. Their power spectrum shows an excess component in the $0.01-0.1 \mathrm{arcmin}^{-1}$ range that cannot be explained by the shot noise of individual galaxies and the power-law spectrum of Galactic cirrus, agreeing with our result. They compared a galaxy clustering model (the dotdashded line in Figure 14) with the measured spectrum and directly derived the bias factor for structure formation in a dark matter universe to be $b \sim 2.4$.

As a result of the comparison of our power spectrum with the Spitzer result, the two spectra in $k<0.1 \operatorname{arcmin}^{-1}$ agree with each other surprisingly well not only in the overall shape of the power law component at very low frequencies by the cirrus and of the galaxy clustering component but also in details such as wiggles at $k=0.01-0.1$ $\operatorname{arcmin}^{-1}$ and a dip around $k \sim 0.02 \operatorname{arcmin}^{-1}$, which may show an oscillatory structure of the baryonic matter distribution. Such concordance between the results of two independent observations with different instruments and in different fields leads us to the conclusion that the clustering components in both AKARI and Spitzer power spectra have the same origin.

It has been reported that galaxy clustering similar to the AKARI and Spitzer results was clearly found by the BLAST experiment in the submillimeter range (Viero et al. 2009). Since the BLAST bands are on the Rayleigh-Jeans side of the dust emission in the rest-frame galaxy, BLAST can be more sensitive to high redshift galaxies than those of AKARI and Spitzer with less contamination by Galactic cirrus. The power spectrum analysis for the BLAST map of $6 \mathrm{deg}^{2}$ area, found a strong signature of galaxy clustering with the power spectrum showing a $k^{2}$-power law over the range of $k \sim 0.03-0.1 \operatorname{arcmin}^{-1}$ in all the BLAST bands. Their power spectra show a very similar shape to those measured by AKARI and Spitzer, in terms of the power-law index of the clustering component and the angular scale at the knee between the shot noise and the clustering components, even though the limiting flux for the source removal for these three missions are different; AKARI $\sim 20 \mathrm{mJy}$, Spitzer $\sim 200 \mathrm{mJy}$, and BLAST 500 mJy.
To extract the clustering component and to compare the AKARI result with the results of other missions on the same basis, we have also made a model fit to our power spectrum using a $k^{-2}$-power law for the clustering component in addition to the shot noise and the cirrus fluctuations, i.e.,

$$
P_{2}(k)=P_{\text {cirrus }}+P_{\text {shot }}+P_{\text {cluster }},
$$

and

$$
P_{\text {cluster }}=P_{c}\left(k / k_{c}\right)^{-2},
$$

where $k_{c}=0.03 \operatorname{arcmin}^{-1}$. Although such a simple model cannot reproduce the complex structure seen in the measured spectrum as described above. For the best-fit case shown by the thin solid line in Figure 14, the amplitude of clustering component is $P_{c}=(1.4 \pm 0.8) \times 10^{3} \mathrm{Jy}^{2} \mathrm{sr}^{-1}$.

The fluctuation amplitude, $\delta I_{\nu}$, of the clustering component relative to the mean CIB level, $<I_{\nu}>$, can be estimated from the power spectrum at a given wavenumber to be Kashlinsky 2005);

$$
\delta I_{\nu}=\sqrt{\left(k^{2} P_{2}(k) / 2 \pi\right)}
$$

Using this relation, our measured fluctuation power of the galaxy clustering $P_{c}$ corresponds to a relative fluctuation amplitude of $\left.\delta I_{\nu} /<I_{\nu}\right\rangle=$ $(2.3 \pm 1.7) \times 10^{-3}$.

It is notable that the CIB distribution at 90 $\mu \mathrm{m}$ after removing the point sources to a faint flux level is unexpectedly smooth. It is also noteworthy that the AKARI data are of high enough quality to detect such very low fluctuation levels.

\subsubsection{SED of the CIB fluctuations}

A study of the SED of the CIB fluctuations should give constraints on the redshift distribution and SED of the underlying galaxies and provide important information on their origin. Figure 15] summarizes the CIB fluctuations measured with AKARI, Spitzer (Lagache et al. 2007) and BLAST (Viero et al. 2009), for shot noise levels at $k=0.5 \operatorname{arcmin}^{-1}$ and for galaxy clustering at $k=0.03 \operatorname{arcmin}^{-1}$, with the fluctuation amplitude $\delta I_{\nu}$. For comparison, the measured mean CIB levels and the model CIB by (Lagache et al. 2004) as in Figure 15] are also shown.

If the clustering components measured by AKARI, Spitzer and BLAST originate from the 


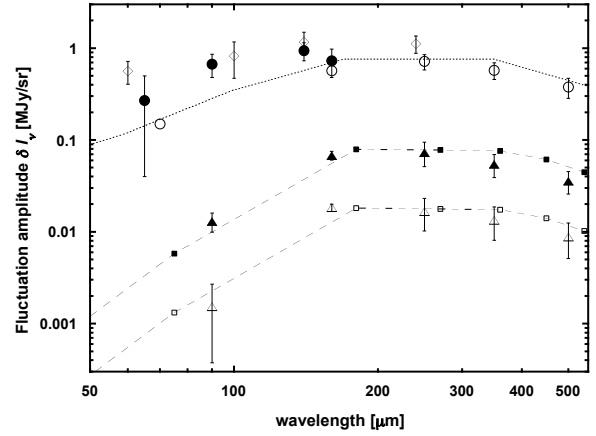

Fig. 15. - The spectral energy distribution (SED) of the CIB fluctuations measured with AKARI at $90 \mu \mathrm{m}$, Spitzer at $160 \mu \mathrm{m}$ (Lagache et al. 2007) and BLAST at 250, 350 and $500 \mu \mathrm{m}$ (Viero et al. 2009) for the shot noise levels at $k=0.5$ $\operatorname{arcmin}^{-1}$ (filled triangles) and the galaxy clustering at $k=0.03 \operatorname{arcmin}^{-1}$ (open triangles). The fluctuation power is converted to linear brightness scale as the fluctuation amplitude, $\delta I(k)=$ $\left.\sqrt{(} k^{2} P_{2}(k) / 2 \pi\right)$. The fluctuations are compared with the absolute CIB levels, $I_{\nu}$, same as in Figure 10. AKARI (filled circles), COBE/DIRBE (diamonds), Spitzer and BLAST (open circles). The model CIB by Lagache et al. (2004) is also indicated (dotted line) as a reference SED of the CIB composed of the $24 \mu \mathrm{m}$ sources, which are stacked with the Spitzer and BLAST data to obtain the mean CIB levels. The dashed lines with data points of the filled squares and the open squares are SED of Arp220 from Klaas et al. (2001), which is redshifted to be at $z=2$ and vertically scaled to fit the CIB fluctuations data for the shot noise (upper) and the galaxy clustering (lower).

same underlying galaxies, the combined spectrum should be comparable to the SED of the most dominant galaxy population. In Figure 15 we compar the combined spectrum of the CIB fluctuations with the SED of Arp220 taken from Klaas et al. 2001), which is redshifted to $z=2$ and scaled to fit the data. Although there is a non-negligible discrepancy between the two spectra, the overall colors are very similar. Therefore, the clustering component is likely to be formed by such luminous infrared galaxies at redshifts of $z>1$, which are all resolved at $24 \mu \mathrm{m}$ and account for $\sim 100 \%$ of the CIB in the submillimeter (Devlin et al. 2009; Pascale et al. 2009; Viero et al. 2009). This result is consistent in that the color of the clustering component is very similar to the color of the CIB in the Spitzer $160 \mu \mathrm{m}$ band and the BLAST bands and also consistent with the mean CIB levels in those bands which agree well with the model CIB for the $24 \mu \mathrm{m}$-selected galaxies.

It is noteworthy that the shot noise as shown in Figure [15] has a very similar spectrum to that of the clustering and to Arp220, in spite of the fact that the shot noise depends strongly on the limiting flux of the point source removal, which are quite different among the missions. This result implies that the brightest underlying galaxies in the field are tracing the clustering structure, because the CIB fluctuation is more sensitive to the brighter end of the source counts than the mean CIB level, as discussed in the previous section.

Another remarkable result on the SED of the CIB fluctuations is that the relative fluctuation amplitude at $90 \mu \mathrm{m}, \delta I_{90} /<I_{90}>$, is quite small compared with those at the other wavelengths, as summarized in Table 5. This is caused by both very red color of the CIB fluctuations and the high CIB level at $90 \mu \mathrm{m}$ exceeding the integrated flux of the $24 \mu \mathrm{m}$-selected galaxies, which can account for the mean CIB levels at longer wavelengths. According to this result, the CIB should have a spectrum peaking around $90 \mu \mathrm{mif}$ it is not to contribute much at $160 \mu \mathrm{m}$ and not to be detected at $24 \mu \mathrm{m}$. The underlying galaxies building the CIB should also be individually faint and numerous to keep the mean CIB levels high while keeping the CIB fluctuations low.

Although hot high- $z$ sources with far-infrared luminosities of the order of $10^{13} L_{\odot}$ discussed in Blain et al. (2004) can considerably contribute to CIB at $90 \mu \mathrm{m}$, such very luminous and less numerous galaxies would largely contribute to the CIB fluctuations rather than the mean CIB brightness. According to the measured CIB properties as discussed above, the bulk CIB may consist of hot high- $z$ sources of lower luminosity but higher surface density. A simple way of reducing the CIB fluctuations without the SED change is to assume higher redshifts and higher temperatures by keeping $T_{d} /(1+z)$ to be a constant, and this could be the case from our result.

To obtain a definite conclusion to the origin of 
the CIB excess in the AKARI band, further investigation is required. To prove the hot high- $z$ source hypothesis, large aperture space telescope missions, such as SPICA (Nakagawa et al. 2008), are required to resolve the far-infrared CIB into point sources to very low flux levels (below $0.1 \mathrm{mJy}$ (Swinvard \& Nakagawa 2009)). Spectral measurement of the continuous SED of the CIB over a wide infrared range would also be a powerful discriminator of the galaxy population. Such observations can be simply done with a small aperture telescopes on small satellites or sub-orbital missions such as rocket experiments. Future space missions to obtain a direct measurement of the midinfrared CIB mitigating the contamination by zodiacal emission require out-of-ecliptic missions or deep space missions beyond the asteroid belt (as the source of zodiacal dust), which will be powerful enough to probe into the underlying galaxies which compose of the CIB excess (Matsuura et al. 2002).

\section{Conclusions}

As the result of a cosmological survey in the ADF-S field, we have successfully measured the absolute sky brightness in the four photometric bands of AKARI at 65, 90, 140 and $160 \mu \mathrm{m}$. AKARI's high sensitivity for diffuse emission, the field selection to minimize the foreground, and careful foreground estimates have enabled us to detect the CIB as an isotropic emission component. The high angular resolution of AKARI was also essential for removing the foreground galaxies as point sources.

The measured CIB brightness in all AKARI bands is consistent with the previous COBE/DIRBE results, and the $90 \mu \mathrm{m}$ brightness is about twice higher than a simple interpolation from the Spitzer results at 70 and $160 \mu \mathrm{m}$ obtained by stacking analysis of $24 \mu \mathrm{m}$-selected sources. The result suggests the exstence of new population(s) with high temperature spectra in addition to the previously explored infrared galaxy population.

By a power spectrum analysis of the $90 \mu \mathrm{m}$ map after removing the point sources down to $\sim 20 \mathrm{mJy}$, we found the shot noise component due to the underlying galaxies. We also detected the signature of galaxy clustering at angular scales of a few tens arcmin. As a result of a comparison of our mea- sured power spectrum with the Spitzer result at $160 \mu \mathrm{m}$ and the BLAST result in the submillimeter range on the same angular scales, the SED of the clustering component is found to be as red as that of ULIRGs at high redshifts, and the main bulk of the CIB may be composed of another population with little contribution to the clustering power.

Our results provide new information on the evolution and the clustering properties of infrared galaxies. To obtain a definite conclusion on the origin of CIB, further investigation is required, e.g., the study of the SEDs of infrared galaxies in detail and a cross-correlation study with multiband images. Resolving the excess brightness into point sources and measuring the CIB spectrum continuously over a wide range would be the main target of on-going and future infrared missions; Herschel, SPICA and other satellites, deep space missions, and sub-orbital programs.

We thank to L. Levenson for his help to calculate the zodiacal foreground in the far-infared based on the model in Wright et al. (1998). This research is based on observations with AKARI, a JAXA project with the participation of ESA. This work was supported by KAKENHI (19540250 and 21111004).

\section{REFERENCES}

Abraham, P., Leinert, C., Acosta-Pulido, J., Schmidtobreick, L., \& Lemke, D. 1998, in The Universe as seen by ISO, ed. P. Cox, \& M. Kessler, ESA SP-427, 145

AKARI FIS Data Users Manual, Version 1.3, 2007 September 14

Blain, A.W. \& Phillips, T.G. 2002, MNRAS, 333, 222

Blain, A.W., Chapman, S.C., Smail, I., \& Ivison, R. 2004, ApJ, 611, 52

Boulanger, F., et al. 1996, A\&A, 312, 256

Bond, J.R., Carr, B., and Hogan, C. 1986, ApJ, 306,428

Chary, R., \& Elbaz, D. 2001, ApJ, 556, 562

Cooray, A., \& Yoshida, N. 2004, MNRAS, 351, L71 
Devlin, M.J., et al. 2009, Nature, 458, 737

Dole, H., et al. 2004, ApJS, 154, 87

Dole, H., et al. 2006, A\&A, 451, 417

Dressler, A. 1980, ApJS, 42, 565

Finkbeiner, D. P., Davis, M., \& Schlegel, D. J. 2000, ApJ, 544, 81

Fixsen, D.J., Dwek, E., Mather, J.C., Bennett, C.L., \& Shafer, R.A. 1998, ApJ, 508, 123

Franceschini, A., Rodighiero, G., \& Vaccari, M. 2008, A\&A, 487, 837

Frayer, D.T., et al. 2006a, AJ, 131, 250

Frayer, D.T., et al. 2006b, ApJ, 647, L9

Hauser, M. G., et al. 1998, ApJ, 508, 25

Hauser, M. G., \& Dwek, E. 2001, ARA\&A, 39, 249

Héraudeau, Ph., et al. 2004, MNRAS, 354, 924

Jeong, W.-S., Lee, H.M., Pak, S., Nakagawa, T., Minn, K.S., Pearson, C.P., \& White, G.J. 2005, MNRAS, 357, 535

Jeong, W.-S., Pearson, C.P., Lee, H.M., Pak, S., \& Nakagawa, T. 2006, MNRAS, 369, 281

Juvela, M., Mattila, K., Lemke, D., Klaas, U., Leinert, C., \& Kiss, Cs. 2009, A\&A, 500, 763

Kalberla, P.M.W., Burton, W.B., Hartmann, D., Amal, E.M., Bajaja, E., Marras, R., \& Pöppel, W.G.L. 2005, A\&A, 440, 775

Kashlinsky, A. 2005, Phys. Rep., 409, 361

Kawada, M., Baba, H., Barthel, P.D., et al. 2007, PASJ, 59, S389

Kawara, K., et al. 1998, A\&A, 336, L9

Kawara, et al. 2004, A\&A, 413, 843

Kelsall, T., et al. 1998, ApJ, 508, 44

Klaas, U., et al. 2001, A\&A, 379, 823

Lagache, G., Haffner, L.M., Reynolds, R.J., Tufte, S.L. 2000a, A\&A, 354, 247
Lagache, G., and Puget, J.-L. 2000b, A\&A, 355, 17

Lagache, G., et al. 2004, ApJS, 154, 112

Lagache, G., Puget, J.-L., \& Dole, H. 2005, ARA\&A, 43, 727

Lagache, G., et al. 2007, ApJ, 665, L89

Levenson, L.L., Wright, E.L., \& Johnson, B.D. 2007, ApJ, 666, 34

Marsden, G., et al. 2009, ApJ, 707, 1729

Matsuhara, H., et al. 2000, A\&A, 361, 407

Matsuhara, H., et al. 2006, PASJ, 58, 673

Matsumoto, T., et al. 2005, ApJ, 626, 31

Matsuura, S. 2002, Proc. Far-IR, Sub-mm \& MM Detector Technology Workshop, Wolf J., Farhoomand J. and McCreight C.R. (eds.), NASA/CP-211408, i-04

Matsuura, S., et al. 2007, PASJ, 59, S503

Miville-Deschênes, M.-A., Lagache, \& G., Puget, J.-L. 2002, A\&A, 393, 749

Miville-Deschênes, M.-A., Lagache, Boulanger, F., \& Puget, J.-L. 2007, A\&A, 469, 595

Nakagawa, T. 2008, Space Telescopes and Instrumentation 2008: Optical, Infrared, and Millimeter. Edited by Oschmann, Jacobus M., Jr.; de Graauw, Mattheus W. M.; MacEwen, Howard A., Proc. SPIE, 7010, 70100H

Papovich, C., Dole, H., Egami, E., et al. 2004, ApJS, 154, 70

Pascale, E., et al. 2009, ApJ, 707, 1740

Pearson, C., et al. 2007, Adv. Sp. Res., 40, 605

Perrotta, F., et al. 2003, MNRAS, 338, 623

Puget, J.-L., Abergel, A., Bernard, J.-P., Boulanger, F., Burton, W.B., Desert, F.-X., \& Hartmann, D. 1996, A\&A, 308, L5

Puget, J.-L., et al. 1999, A\&A, 345, 29

Reach, W. T. 1988, ApJ, 335, 468 
Reach, W. T., Wall, W. F. \& Odegard, N. 1998, ApJ, 507, 507

Rodighiero, G., Lari, C., Franceschini, A., Gregnanin, A., \& Fadda, D. 2003, MNRAS, 343, 1155

Santos, M.R., Bromm, V., \& Kamionkowski, M. 2002, MNRAS, 336, 1082

Schlegel, D. J., Finkbeiner, \& D. P., Davis, M. 1998, ApJ, 500, 525

Serjeant, S., et al. 2004, MNRAS, 355, 813

Shirahata, M., et al. 2008, in ASP Conf. Ser. 399, Panoramic Views of Galaxy Formation and Evolution, ed. T. Kodama, T. Yamada, \& K. Aoki (San Francisco, CA: ASP), 290

Shirahata, M., et al. 2009, PASJ, 61, 737

Swinyard, B., \& Nakagawa, T. 2009, Exp. Astron., 23, 193

Takeuchi, T. T., \& Ishii, T. T. 2010, Adv. Sp. Res., submitted

Treister, E., et al. 2006, ApJ, 640, 603

Viero, M. P., et al. 2009, ApJ, 707, 1766

Wall, J. V., Pope, A., \& Scott, D. 2008, MNRAS, 383,435

Wright, E. 1998, ApJ, 496, 1

Wright, E. L. 2001, IAUS, 204, 157

This 2-column preprint was prepared with the AAS LATEX macros v5.2. 
Table 1: Observation log.

\begin{tabular}{ccccc}
\hline \hline Field & Center coord. (J2000) & Observation date & AOT(parameter) & $N_{o b s}$ \\
\hline ADF-S & $4^{h} 44^{m} 00^{s},-53^{\circ} 20^{\prime} 00^{\prime \prime}$ & 2006 Jul - 2006 Aug & FIS-02(2.0;15;1) & 53 \\
ADF-S & $4^{h} 44^{m} 00^{s},-53^{\circ} 20^{\prime} 00^{\prime \prime}$ & 2007 Jan - 2007 Feb & FIS-02 $(2.0 ; 15 ; 1)$ & 63 \\
ADF-S & $4^{h} 44^{m} 00^{s},-53^{\circ} 20^{\prime} 00^{\prime \prime}$ & 2007 Jul - 2007 Aug & FIS-01 $(1.0 ; 15 ; 1)$ & 84 \\
LH-PVV $^{\mathrm{a}}$ & $10^{h} 50^{m} 36^{s}, 57^{\circ} 27^{\prime} 09^{\prime \prime}$ & 2006 May & FIS-02 $(2.0: 15 ; 1)$ & 11 \\
LH-MP $^{\mathrm{b}}$ & $10^{h} 50^{m} 25^{s}, 57^{\circ} 36^{\prime} 37^{\prime \prime}$ & 2007 May & FIS-01 $(2.0 ; 08 ; 1)$ & 3 \\
NEP-DT $^{c}$ & $17^{h} 55^{m} 24^{s}, 66^{\circ} 37^{\prime} 32^{\prime \prime}$ & 2007 Oct & FIS-01 $(2.0 ; 08 ; 1)$ & 4 \\
\hline
\end{tabular}

${ }^{a}$ Performance verification (PV) phase observations

${ }^{b}$ Mission program (MP) observations

${ }^{c}$ Director time (DT) observations 
Table 2: Summary of foregrounds and mean CIB levels in ADF-S

\begin{tabular}{cccccc}
\hline \hline $\begin{array}{c}\text { Wavelength } \\
(\mu \mathrm{m})\end{array}$ & $\begin{array}{c}\text { Total }^{\mathrm{a}} \\
\left(\mathrm{MJy} \mathrm{sr}^{-1}\right)\end{array}$ & $\begin{array}{c}\text { Zodiacal }^{\mathrm{b}} \\
\left(\mathrm{MJy} \mathrm{sr}^{-1}\right)\end{array}$ & $\begin{array}{c}\text { Cirrus }^{\mathrm{c}} \\
\left(\mathrm{MJy} \mathrm{sr}^{-1}\right)\end{array}$ & $\begin{array}{c}\mathrm{CIB}^{\mathrm{d}} \\
\left(\mathrm{MJy} \mathrm{sr}^{-1}\right)\end{array}$ & $\begin{array}{c}\text { Models }^{\mathrm{e}} \\
\left(\mathrm{MJy} \mathrm{sr}^{-1}\right)\end{array}$ \\
\hline 65 & $4.31 \pm 0.04$ & 3.97 & 0.09 & $0.27 \pm 0.03 \pm 0.20$ & $0.1-0.2$ \\
90 & $3.57 \pm 0.02$ & 2.72 & 0.22 & $0.67 \pm 0.05 \pm 0.14$ & $0.2-0.5$ \\
140 & $2.36 \pm 0.16$ & 0.90 & 0.57 & $0.94 \pm 0.16 \pm 0.05$ & $0.5-1.4$ \\
160 & $2.02 \pm 0.20$ & 0.77 & 0.60 & $0.73 \pm 0.21 \pm 0.04$ & $0.5-1.6$ \\
\hline
\end{tabular}

${ }^{a}$ Mean sky brightness of all observations derived with the in-flight calibration factor. The error denotes statistical error only. ${ }^{b}$ Mean zodiacal brightness of all observations calculated with the zodiacal emission model (Kelsall et al. 1998).

${ }^{c}$ Mean cirrus brightness in the entire field converted from SFD map at $100 \mu \mathrm{m}$ (Finkbeiner et al. 2000) by using the linear correlation for the short wavelength bands and assuming the blackbody spectrum with $T=18.2 \mathrm{~K}$ and $n=2$ for the long wavelength bands.

${ }^{d}$ In the short wavelength bands, zero-intercept of linear correlation between AKARI and SFD maps to subtract cirrus brightness. In the long wave bands, the mean cirrus brightness was subtracted from the zodi-subtracted mean brightness. The gain factor measured in the laboratory is used. The first error includes both statistical error and calibration error, and the second error is uncertainty of the zodi-model.

${ }^{e}$ CIB brightness range predicted from galaxy evolution models, Lagache et al. (2004); Pearson et al. (2007); Franceschini et al. (2008); Takeuchi \& Ishii (2010). 
Table 3: Field variance of CIB brightness

\begin{tabular}{cccccc}
\hline \hline Field & $\begin{array}{c}N_{H I^{\mathrm{a}}} \\
\left(10^{20} \mathrm{~cm}^{-2}\right)\end{array}$ & $\begin{array}{c}I_{65}{ }^{\mathrm{b}} \\
\left(\mathrm{MJy} \mathrm{sr}^{-1}\right)\end{array}$ & residual $^{\mathrm{c}}$ & $\begin{array}{c}I_{90}{ }^{\mathrm{b}} \\
\left(\mathrm{MJy} \mathrm{sr}^{-1}\right)\end{array}$ & residual $^{\mathrm{C}}$ \\
\hline ADF-S & 0.67 & $0.329 \pm 0.003$ & $0.21 \pm 0.02$ & $0.835 \pm 0.004$ & $0.54 \pm 0.04$ \\
LH-MP & 0.54 & $0.311 \pm 0.057$ & $0.22 \pm 0.06$ & $0.873 \pm 0.021$ & $0.64 \pm 0.04$ \\
NEP-DT & 3.73 & $0.986 \pm 0.023$ & $0.33 \pm 0.09$ & $2.038 \pm 0.058$ & $0.42 \pm 0.22$ \\
average & & & $0.22 \pm 0.02$ & & $0.59 \pm 0.03$ \\
\hline
\end{tabular}

${ }^{a}$ Taken from the LAB HI $20 \mathrm{~cm}$ survey (Kalberla et al. 2005).

${ }^{b}$ The mean brightness after subtraction of the zodiacal foreground with the errors estimated from the variance for a number of observations in each field.

${ }^{c}$ Obtained by subtraction of the HI-correlated component, assuming the conversion factor at $100 \mu \mathrm{m}$ to be $0.6 \pm 0.1 \mathrm{MJy}$ $\mathrm{sr}^{-1} / 10^{20} \mathrm{~cm}^{-2}$ and extrapolating it to each wavelength by the blackbody spectrum with $T=18.2 \mathrm{~K}$ and $n=2$. 
Table 4: Small-scale fluctuations of foregrounds and the CIB ${ }^{\text {a }}$

\begin{tabular}{ccccc}
\hline \hline Zodiacal $^{\mathrm{b}}$ & Cirrus $^{\mathrm{C}}$ & ${\text { CIB } P_{\text {shot }}{ }^{\mathrm{d}}}$ & Models $^{\mathrm{e}}$ & ISO $^{\mathrm{f}}$ \\
\hline$<0.6 \times 10^{2}$ & $<0.1 \times 10^{2}$ & $3.6 \pm 0.2 \times 10^{2}$ & $(1.1-7.2) \times 10^{3}$ & $2 \times 10^{4}$ \\
\hline
\end{tabular}

${ }^{a}$ Power pectral density at $90 \mu \mathrm{m}$ in $\mathrm{Jy}^{2} \mathrm{sr}^{-1}$.

${ }^{b}<1 \%$ of the mean brightness at angular scale of 1 arcmin (Abraham et al. 1998).

${ }^{c}$ Cirrus fluctuation power at $k>0.2 \mathrm{arcmin}^{-1}$ estimated from the measured $P_{0}$.

${ }^{d}$ The mean shot noise level in $k=0.2-0.8 \operatorname{arcmin}^{-1}$ after point-source removal.

${ }^{e}$ Galaxy evolution models, Lagache et al. (2004); Jeong et al. (2006); Pearson et al. (2007); Franceschini et al. (2008); Takeuchi \& Ishii (2010).

${ }^{f}$ ISO result at $90 \mu \mathrm{m}$ after removing galaxies brighter than $30 \mathrm{mJy}$, Matsuhara et al. (2000). 
Table 5: Measured SED of the clustering component of the CIB fluctuations

\begin{tabular}{cccc}
\hline \hline Wavelength $(\mu \mathrm{m})$ & $\delta I_{\nu}\left(\mathrm{MJy} \mathrm{sr}^{-1}\right)$ & $\delta I_{\nu} /<I_{\nu}>$ & Reference \\
\hline 90 & $(1.5 \pm 1.1) \times 10^{-3}$ & $(2.3 \pm 1.7) \times 10^{-3}$ & This work \\
160 & $(1.8 \pm 0.2) \times 10^{-2}$ & $(3.2 \pm 0.3) \times 10^{-2}$ & 1,2 \\
250 & $(1.7 \pm 0.3) \times 10^{-2}$ & $(2.3 \pm 0.4) \times 10^{-2}$ & 3,4 \\
350 & $(1.3 \pm 0.3) \times 10^{-2}$ & $(2.3 \pm 0.5) \times 10^{-2}$ & 3,4 \\
500 & $(0.9 \pm 0.2) \times 10^{-2}$ & $(2.3 \pm 0.6) \times 10^{-2}$ & 3,4 \\
\hline
\end{tabular}

Notes. The fluctuation amplitude of the clustering component estimated from Eq. 6 for $k=0.03 \operatorname{arcmin}^{-1}$.

References. (1) Dole et al. (2006); (2) Lagache et al. (2007); (3) Marsden et al. (2009); (4) Viero et al. (2009) 